BGPE Discussion

Paper No. 201

\title{
Multidimensional Noise and Non-Fundamental Information Diversity
}

\section{David Russ}

December 2020

\section{ISSN 1863-5733}

Editor: Prof. Regina T. Riphahn, Ph.D.

Friedrich-Alexander-Universität Erlangen-Nürnberg

(c) David Russ 


\title{
Multidimensional Noise and Non-Fundamental Information Diversity
}

\author{
David Russ \\ University of Regensburg, Germany
}

December 14, 2020

\begin{abstract}
In this paper, I relax the common assumption of the one-dimensionality of noise made in the standard competitive noisy rational expectations framework. Within an environment characterized by multidimensional noise, I explore the strategic interactions between different traders that are informed about different components of the noise inherent in the market price. I find that agents' trades against different types of noise are complements due to an inference augmentation effect. As one group trades more aggressively against the part of the noise they observe, the market price becomes a more precise signal for fundamentals for the other noise-informed groups. Since traders use their information about noise together with the market price in order to infer information about fundamentals, this makes the other groups trade more aggressively against their observed piece of noise, too. Strategic complementarities can also be found in the information market. Both acquiring information about the same type and about different types of noise can be complements.
\end{abstract}

JEL Classifications: C62, D53, G12, G40.

Keywords: Rational Expectations Equilibrium, Complementarities, Noise Trading, Non-Fundamental Information. 


\section{Introduction}

Recently, retail investment has experienced an era of immense growth. As of 2020, retail investment is responsible for $25 \%$ of total trading volume in US equity markets, compared to $10 \%$ in 2019 according to the Traders Magazine. ${ }^{1}$ Due to a lack of investment alternatives caused by the current low-interest phase in most industrialized countries, private households have turned their attention to the stock market in search for returns on their savings. This increased trading activity by retail investors has significantly contributed to a boom in the online brokerage sector. In their competition for the growing mass of customers, online brokers have significantly reduced their commissions. The often-debated trading application Robinhood, e.g., tries to attract clients by promising zero trading fees. As reported by $C N B C$, this offer has made more than three million retail investors open an account via Robinhood, only in the first four month of $2020 .^{2}$

Ever since Milton Friedman, it its well known that there is no "free lunch" in economies. Instead of paying with money, customers of commission-free trading applications pay with data. Robinhood and other trading applications mainly make money by passing on their customer orders to third parties in the market. This method is known as "payment for order flow" (PFOF). The third parties, then, can try to match the order flows with other suitable ones and profit from the bid-ask spread.

Thus, the recent boom in retail investment has not only lead to a growth in the online brokerage sector, but also to an increase in available information about retail investors' orders in financial markets. As retail investment is responsible for a remarkable size of total trading volume, such information is not only valuable when trying to match orders, but also when trading on one's own account. More particularly, due to PFOF, financial markets are populated by different traders who observe different pieces of the whole order flow linked to retail investment in the market. The aim of this paper is to analyze the interactions that emerge between these diverse informed traders.

In the academic literature, retail investors are often referred to as "noise traders", i.e., unsophisticated, inexperienced traders, whose orders are uncorrelated to fundamentals (see, e.g., Black (1986) and Barber et al. (2006)). Interpreting retail investors as noise traders makes the competitive noisy rational expectations framework à la Grossman \& Stiglitz (1980) (henceforth: GS 1980) and Hellwig (1980) a suitable

\footnotetext{
${ }^{1}$ Shanny Basar, "Retail Investing Evolves", Traders Magazine, September 2020, https://www. tradersmagazine.com/am/retail-investing-evolves.

${ }^{2}$ Weizhen Tan, "Retail investing boom may spark concerns", CNBC, September 2020, https://www. cnbc. com/2020/09/10/markets-have-lived-through-retail-investing-booms -before-strategist-says.html.
} 
framework for the analysis of the expounded research task. Nevertheless, there is one important modification that has to be made compared to the standard framework. The vast majority of the existing literature assumes noise to be one-dimensional. ${ }^{3}$ The demand coming from noise traders, which prevents the price from fully revealing fundamentals in equilibrium is summed up in a single component. This common assumption, however, does not account for the fact that there can exist sophisticated traders in the market who possess unbiased knowledge of a part of the whole demand stemming from noise traders. That is, rather than gleaning information about the whole order flow linked to noise trading, these investors precisely know the demand of some noise traders in the market. Of course, there exist other noise traders, whose orders they do not observe. Thus, we need to extend the noisy rational expectations framework to the case where noise is not one-, but multidimensional. This means that the market price is shaped by more than one noise factor in equilibrium.

In a static competitive economy, agents use their information about noise, i.e., their non-fundamental information to extract noise from the market price and to gain a more precise signal about fundamentals out of it. Whenever non-fundamentally informed traders observe a high noise trader demand, they expect the price to be very noisy and, thus, fundamentals to be very low, which makes them reduce their demand. Inversely, if they observe low noise trader demand, fundamentals are expected to be high and they raise their demand as a consequence. That is, rational agents follow a contrarian strategy with respect to their non-fundamental information. They trade against noise traders and, therefore, mitigate their influence on prices.

The central question concerning the financial market is how agents' trades against different noise trader demands are connected. Does more aggressive trading against noise by one group encourage other groups to trade more or less aggressively against the type of noise that they observe? When looking at the information market, we aim at exploring two different types of interactions in information acquisition. On the one hand, we would like to assess whether acquiring information about the same type of noise is a complement or a substitute. This type of interaction could also be studied in an environment characterized by one-dimensional noise. On the other hand, when noise is multidimensional, one can also investigate whether acquiring information about different types of noise is a complement or a substitute. Thus, multidimensionality of noise allows to assess whether cross-complementarities or cross-substitutabilities in non-fundamental information acquisition exist.

I find that both the financial market and the information market are characterized by complementarities. It is worth stressing that complementarities do not arise per se in this type of models. The seminal model of GS 1980 is characterized by strategic substitutability in (fundamental) information acquisition. At the trading

\footnotetext{
${ }^{3}$ One of the very few exceptions constitutes, e.g., Gennotte \& Leland (1990).
} 
stage, more aggressive trading against noise by one group encourages other ones to do the same. This is due to an inference augmentation effect. More aggressive trading against one type of noise makes the market price react less to it relative to fundamentals. Hence, all traders that do not observe this specific type of noise profit from a more informative price signal. As a consequence, they trade more aggressively on it. Since all noise-informed agents use their non-fundamental information together with the market price to infer information about fundamentals, more aggressive trading on their price signal implies more aggressive trading against the type of noise they observe. This strong complementarity in trading leads to the existence of multiple equilibria in financial markets, that show, if noise is two-dimensional, similar properties to those of Ganguli \& Yang (2009). If noise is three-dimensional, these properties significantly differ from the two-dimensional case.

In the information market, I can show that both acquiring information about the same type and about different types of noise can be complements. These results are driven by the fact that a rise in the mass of one noise-informed group does not only affect how this group but also how other non-fundamentally informed ones trade against the pieces of noise that they observe. Thus, more non-fundamentally informed traders do not only affect price informativeness as a whole but also the uncertainty each specific noise-informed group faces (which crucially depends on how other noise-informed groups trade against their observed type of noise).

By contrast, in the absence of different noise-informed groups, acquiring information about the same of type of noise is always a substitute. In the reduced setup with only one noise-informed group, a rise in the mass of this group makes the price more informative about fundamentals, but does not affect how other pieces of noise influence the market price. As a consequence, the uncertainty the already noise-informed traders face does not change, which lowers the incentive for others to acquire non-fundamental information. Complementarities only occur with different noise-informed groups. Moreover, I show that multiple equilibria can also exist in the information market.

My results relate to two growing strands of the literature concerning the competitive noisy rational expectations framework. The first strands deals with the effects of nonfundamental information. The pioneering model dates back to Gennotte \& Leland (1990). In their specification, a fixed portion of traders observes a part of the noisy asset supply, which is driven by liquidity traders. The authors focus on explaining stock market crashes that can occur due to unobserved shifts in supply, i.e., due to a lack of non-fundamental information. Manzano \& Vives (2011) consider a static economy where rational traders receive private information about the noisy asset supply. The existence of private non-fundamental signals can lead to self-fulfilling equilibria in the financial market as it opens up new possibilities for the coordination 
among traders that do not in exist in the original framework.

Cespa \& Vives (2015) assume correlated noise shocks in an overlapping-generations model with two trading periods. If noise shocks across periods are correlated, traders can use the current price in order to infer information about the current noise shock, which then yields information about next period's noise. Persistent noise trading generates multiple equilibria in financial markets as it induces strategic complementarities among short-term investor in the use of their private fundamental information. They identify an equilibrium that is characterized by high price informativeness. This finding challenges the widespread view that short-term trading harms price informativeness. In a closely related framework, Cespa \& Vives (2012) show that persistent noise trading can also generate multiple equilibria and improve price informativeness in an economy with long-term investors.

Marmora \& Rytchkov (2018) lay the focus on the impact of non-fundamental information acquisition. In an economy where agents are endowed with diverse prior information about the fundamental asset value, they assign each rational trader a fixed information processing capacity that they can use to produce private fundamental and private non-fundamental information. They show that agents tend to specialize in information acquisition. Those with precise prior information about fundamentals focus on the acquisition of fundamental information, those with imprecise prior information switch to acquiring non-fundamental information. Moreover, the existence of non-fundamental information increases price informativeness although it can crowd out fundamental information.

Along similar lines, Farboodi \& Veldkamp (2020) analyze the effects coming from nonfundamental information acquisition in an infinite-horizon economy that is populated by overlapping generations They assign traders a data constraint to process contemporaneous private fundamental and contemporaneous private non-fundamental information. There is technological progress over time., i.e., agents can process more information as time advances. When technology is rather poor, investors focus on producing fundamental information. As technology improves, they begin to produce non-fundamental information. As technology becomes very sophisticated, both types of information become abundant. Analogously to Marmora \& Rytchkov (2018), they show that non-fundamental information also increases price informativeness in a dynamic context.

Second, this paper relates to the strand of the literature that aims at showing that traders' interactions can be characterized by complementarities in a competitive trading environment. In a dynamic three-period setting with long-lived agents, Hirshleifer et al. (1994) show that complementarities in fundamental information acquisition can occur if there are traders that receive private fundamental information early than other ones. In Veldkamp (2006), the cost of acquiring fundamental information is en- 
dogenous and negatively depends on the mass of informed traders. This relationship can induce complementarities in information acquisition. Garcia \& Strobl (2011) study a setup where agents derive utility from comparing their wealth to the average wealth in the economy. More informed trading increases average wealth, which then can increase the incentive for uninformed traders to acquire information. In Rahi \& Zigrand (2014), complementarities are induced by different private evaluations of the value of an asset. Goldstein et al. (2014) show that complementarities can occur if agents' investment opportunities differ.

In a dynamic setup with two trading periods and long-lived agents, Avdis (2016) shows that acquiring fundamental information can be a complement if noise shocks across periods are correlated. Since first-period agents use the first-period price to infer information about next period's noise shock, more fundamentally informed trading makes it more difficult to glean this type of information by disentangling the price. As a consequence, the incentive for uninformed traders to acquire fundamental information increases since fundamental information helps them to extract non-fundamental information from the market price.

In a closer related setup to mine, Ganguli \& Yang (2009) show within an environment characterized by one-dimensional noise that the existence of private non-fundamental information can lead to complementarities in fundamental information acquisition. The reason for this is that more fundamentally informed trading can make prices less informative about fundamentals in the presence of private non-fundamental information. As more fundamentally informed traders enter the market, the price can become less informative, which increases the incentive for others to acquire fundamental information. Moreover, they show that acquiring a fixed bundle of private fundamental and private non-fundamental information can be a complement.

In a methodologically related paper, Goldstein \& Yang (2015) (henceforth: GY (2015)) extend the seminal model of GS 1980 by assuming different traders that are informed about different fundamentals, that jointly determine the "fair" value of a stock. They show that different agents' trades on different fundamentals can be complements due to an uncertainty reduction effect. As one group of fundamentally informed traders trades more aggressively on the fundamental they know, the uncertainty the other traders face is reduced. This makes them trade more aggressively on their own fundamental information, too.

However, GY (2015, p. 1733) identify a second effect, an "inference augmentation effect", which favors strategic substitutability in trading in their setup. As one informed group trades more aggressively on their fundamental, the price becomes more informative about this fundamental for traders that do not observe it. Hence, they rely more on the market price when predicting the unknown fundamental. Since a higher value for the fundamental they observe ceteris paribus indicates a 
lower value for the unobserved fundamental when looking at the market price, they reduce their demand for the risky asset when their observed fundamental takes on a higher value. The higher the informativeness of the market price about the unknown fundamental (which crucially depends on how aggressively the other group trades on their fundamental information), the more they lower their demand as a reaction to observing a higher value of the fundamental they know.

When concerning diverse non-fundamental information, I also identify an inference augmentation effect, that, however, works in the opposite direction, i.e., it induces complementarities in trading. While this effect prevents equilibrium multiplicity in GY 2015 by leading to substitutabilities in trading, it is responsible for generating multiple equilibria in this paper.

Although fundamental information is diverse, acquiring information about the same fundamental is always a substitute in GY 2015. However, they show that acquiring information about different fundamentals can be a complement. As already mentioned, non-fundamental information acquisition is characterized by possible complementarities in both respective types.

The remainder of this paper is structured as follows: Section 2 describes the model and derives its equilibrium in the financial market. Section 3 focuses on traders' interaction at the trading stage. Section 4 derives the equilibrium in the information market and explores the respective interactions therein. Section 5 shortly discusses the results of an extension with three-dimensional noise. Section 6 concludes.

\section{The Model}

\section{A. Model Assumptions}

The financial market consists of one riskless and one risky asset. The riskless asset (i.e., a bond) can be traded without supply restrictions. Its return is normalized to zero and it serves as a numeraire in the economy. The risky asset (i.e., a stock) in zero net supply is traded at market price $P$ in the first period. In the second period, it pays off its fundamental value given by $\theta \sim \mathrm{N}\left(0, \tau_{\theta}^{-1}\right)$.

There are six different types of agents in the financial market, two of which stand for noise traders with demand $x_{1} \sim \mathrm{N}\left(0, \tau_{x}^{-1}\right)$ and $x_{2} \sim \mathrm{N}\left(0, \tau_{x}^{-1}\right)$, respectively. ${ }^{4}$ Moreover, there exist two sets of non-fundamentally informed agents indexed by $\left[0, \lambda_{1}\right]$ and by $\left[0, \lambda_{2}\right]$, respectively. Each trader $n_{1} \in\left[0, \lambda_{1}\right]$ observes $x_{1}$ whereas each trader $n_{2} \in\left[0, \lambda_{2}\right]$ knows $x_{2}$. Additionally, there is a continuum of fundamentally informed

\footnotetext{
${ }^{4}$ One could also interpret each component of noise trader demand as the sum of a fixed number of single noise trader demands.
} 
traders indexed by the interval $[0,1] .{ }^{5}$ Each trader $f \in[0,1]$ observes $s_{f}=\theta+\epsilon_{f}$, where $\epsilon_{f} \sim$ i.i.d. $\mathrm{N}\left(0, \tau_{\epsilon}^{-1}\right)$. There is also a continuum of completely uninformed but rational traders indexed by the interval $\left[0, \lambda_{u}\right]$. Each trader $u \in\left[0, \lambda_{u}\right]$ only observes the market price. $\theta, x_{1}, x_{2}$, and $\epsilon_{f}$ are assumed to be pairwise independent for all $f$. Since the market is assumed to competitive, all agents are price takers. Therefore, they (additionally) use the market price in order to infer information about $\theta$. For $k=n_{1}, n_{2}, f, u$, agent $k$ 's final wealth is given by $\pi_{k}=(\theta-P) D_{k}$, where $D_{k}$ stands for agent $k$ 's demand for the risky asset. Without the loss of generality, we normalize agents' initial wealth to zero. All rational traders are characterized by a negative exponential utility function with constant absolute risk aversion (CARA), $U\left(\pi_{k}\right)=-e^{-\gamma \pi_{k}} \cdot \gamma(>0)$ measures agents' common degree of risk aversion.

\section{B. Equilibrium Determination}

In the spirit of GS 1980, the equilibrium that we derive is a rational expectations equilibrium (REE). The price function is determined by a conjecture-and-verify approach. Suppose that the price is linear in the state variables, i.e.,

$$
P=a_{\theta} \theta+a_{1} x_{1}+a_{2} x_{2} .
$$

All rational agents aim at maximizing their expected utility conditional on the information that they observe by optimally choosing their demand for the risky asset. Maximizing their CARA utility function yields

$$
D_{k}=\frac{\mathbb{E}\left[\theta \mid \mathcal{F}_{k}\right]-P}{\gamma \operatorname{Var}\left[\theta \mid \mathcal{F}_{k}\right]}
$$

where $\mathcal{F}_{k}$ stands for agent $k$ 's information set. We have $\mathcal{F}_{n_{1}}=\left\{P, x_{1}\right\}, \mathcal{F}_{n_{2}}=\left\{P, x_{2}\right\}$, $\mathcal{F}_{f}=\left\{P, s_{f}\right\}$, and $\mathcal{F}_{u}=\{P\}$. Intuitively, traders go long (resp., short) in the risky asset if the expected value of $\theta$ exceeds (resp., is inferior to) the market price. Because of their risk aversion, demand is constrained. The higher the uncertainty about fundamentals or the higher the degree of risk aversion, the lower is the demand in absolute terms.

The non-fundamentally informed agents use their knowledge about noise trader demand to generate a more precise signal for the fundamental asset value out of the market price. Building on (1), the price turns into the following signal for the $x_{1}$-informed traders, which they use to update their prior expectations about $\theta$ :

$\hat{P}_{\mathrm{n}_{1}} \equiv \frac{P-a_{1} x_{1}}{a_{\theta}}=\theta+\frac{1}{\beta} x_{2}$,

\footnotetext{
${ }^{5}$ Any mass different from unity would leave all derived results unchanged.
} 
with $\beta \equiv a_{\theta} / a_{2}$. Analogously, the $x_{2}$-informed ones can disentangle the market price as follows:

$\hat{P}_{\mathrm{n}_{2}} \equiv \frac{P-a_{2} x_{2}}{a_{\theta}}=\theta+\frac{1}{\alpha} x_{1}$,

with $\alpha \equiv a_{\theta} / a_{1}$. Thus, for the non-fundamentally informed traders, the market price is a signal about $\theta$ with precision $\beta^{2} \tau_{x}$ and $\alpha^{2} \tau_{x}$, respectively. By (3) and (4), it can be seen that a rise in $x_{1}$ or $x_{2}$ reduces the demand of the respective noise-informed group. The explanation for this is the following: As the noise-informed agents use their knowledge about noise trader demand to gain a more precise signal about $\theta$ out of the market price, their signal (i.e., $\hat{P}_{\mathrm{n}_{1}}$ or $\hat{P}_{\mathrm{n}_{2}}$ ) ceteris paribus indicates a lower value for the risky fundamental asset value if $x_{1}$ and $x_{2}$ go up, respectively. Due to a lower expected fundamental value, the noise-informed agents reduce their demand. Thus, they follow a contrarian strategy with respect to their information about noise. The more precise their signal gained from decomposing the market price is, the more they reduce their demand as a response to a rise in noise trader demand.

For the fundamentally informed agents as well as for the completely uninformed ones, observing the price is informationally equivalent to observing

$\hat{\mathrm{P}}_{\mathrm{f} / \mathrm{u}} \equiv \frac{P}{a_{\theta}}=\theta+\frac{a_{1} x_{1}+a_{2} x_{2}}{a_{\theta}}$.

Hence, $\hat{\mathrm{P}}_{\mathrm{f} / \mathrm{u}}$ is a signal about $\theta$ with precision $\tau_{x} /\left(1 / \alpha^{2}+1 / \beta^{2}\right)$. Without nonfundamental information, the signal for $\theta$ generated by disentangling the market price clearly has a lower precision. Using (3), (4), (5), and agents' private fundamental signals, the first two conditional moments of $\theta$ can be determined for all types of traders by using the projection theorem.

Then, the price $P$ is determined by clearing the asset market:

$\int_{0}^{1} \mathrm{D}_{\mathrm{f}} \mathrm{df}+\int_{0}^{\lambda_{1}} \mathrm{D}_{\mathrm{n}_{1}} \mathrm{dn}_{1}+\int_{0}^{\lambda_{2}} \mathrm{D}_{\mathrm{n}_{2}} \mathrm{dn}_{2}+\int_{0}^{\lambda_{u}} \mathrm{D}_{\mathrm{u}} \mathrm{du}+x_{1}+x_{2}=0$.

By plugging rational agents' demand function from (2) into (6), one can solve for $P$ and show that it is indeed determined by a linear function of the state variables as conjectured in (1). After matching coefficients, we obtain the following function of $P$ in the REE (the proof and the definition of $\Delta_{\beta}$ can be found in the Appendix):

Proposition 1. If $\Delta_{\beta}<0$ (resp., $\Delta_{\beta}=0$ ), there exist(s) two (resp., one) partially revealing $R E E$ given by

$P=a_{\theta} \theta+a_{1} x_{1}+a_{2} x_{2}$, 
where

$a_{\theta}=\frac{\alpha^{4} \lambda_{2} \tau_{x}+\beta^{2}\left(\tau_{\epsilon}+\lambda_{1} \beta^{2} \tau_{x}\right)+\alpha^{2}\left(\tau_{\epsilon}+\beta^{2} \tau_{x} \omega\right)}{\alpha^{4} \lambda_{2} \tau_{x}+\beta^{2}\left(\tau_{\epsilon}+\lambda_{1} \beta^{2} \tau_{x}+\tau_{\theta} \omega\right)+\alpha^{2}\left[\tau_{\epsilon}+\left(\beta^{2} \tau_{x}+\tau_{\theta}\right) \omega\right]}$,

$a_{1}=\frac{1}{\alpha} a_{\theta}$,

$a_{2}=\frac{1}{\beta} a_{\theta}$,

$\omega \equiv 1+\lambda_{1}+\lambda_{2}+\lambda_{u}$

and $\alpha$ as well as $\beta$ are solutions of the following fixed-point equations:

$\alpha=f(\alpha) \equiv \frac{\lambda_{1} \lambda_{2}^{2} \tau_{x}^{3} \alpha^{4}+2 \lambda_{1} \lambda_{2} \tau_{x}^{2} \tau_{\epsilon} \alpha^{2}+\tau_{\epsilon}\left(\lambda_{1} \tau_{x} \tau_{\epsilon}+\gamma^{2}\right)}{\gamma^{3}}$,

$\beta=f(\beta) \equiv \frac{\lambda_{1}^{2} \lambda_{2} \tau_{x}^{3} \beta^{4}+2 \lambda_{1} \lambda_{2} \tau_{x}^{2} \tau_{\epsilon} \beta^{2}+\tau_{\epsilon}\left(\lambda_{2} \tau_{x} \tau_{\epsilon}+\gamma^{2}\right)}{\gamma^{3}}$.

According to proposition 1, there are, apart from one combination of the exogenous parameters that yields $\Delta_{\beta}=0$, two REE if an equilibrium exists. The number of equilibria is pinned down by the number of solutions for $\alpha$ and $\beta$. Since their solutions are hardly analytically tractable, we illustrate them numerically. Figure 1 shows the mapping of $f(\beta)$ with $\beta$ where $\gamma=1, \tau_{\epsilon}=0.3, \tau_{x}=0.5, \lambda_{1}=0.6$, and $\lambda_{2}=0.5$.

The two intersections of the graph with the dashed $45^{\circ}$-line mark the two equilibria

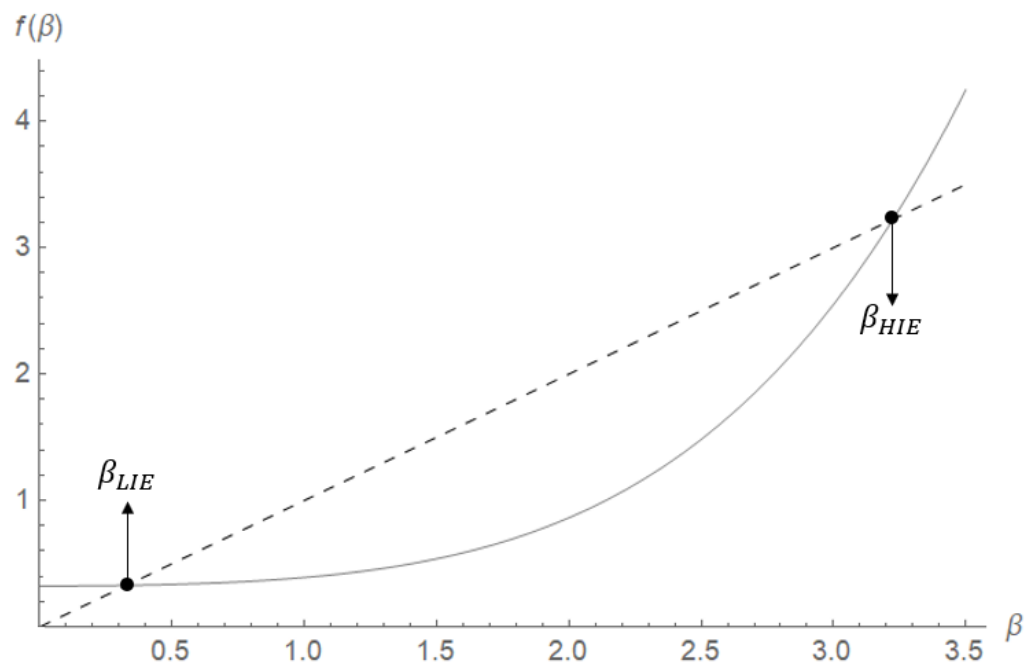

Figure 1: Mapping $f(\beta)$ with $\beta$.

of the model. From the definitions of $\alpha$ and $\beta$, it can be concluded that they measure 
how strongly the price reacts to the fundamental asset value relative to the respective noise shock. Since high (resp., low) values of $\beta$ and $\alpha$ imply that the market price is mainly driven by fundamental information (resp., by noise), we refer to the first intersection in Figure 1 as the low information equilibrium (LIE) and to the second one as the high information equilibrium (HIE). In the next section, some further conditions that ensure the existence of an equilibrium are derived.

\section{Interactions at the Trading Stage}

Now, we turn to the interactions of the diverse non-fundamentally informed groups at the trading stage. Particularly, we are interested in how their trades against the two different noise shocks are connected. Moreover, we would like to analyze the effects of their interactions on price informativeness and assess what effects a rise in the mass of non-fundamentally informed traders exerts on the equilibria of the financial market.

\section{A. Trading Intensities}

The term "trading intensity" refers to the amount of aggressiveness with which the noise-informed traders trade against their signal about noise trader demand. Hence, it measures how much noise is actually counteracted by the rational, nonfundamentally informed agents. Since there are two different groups of noise-informed traders, we deal with two trading intensities. From the demand functions of the noise-informed traders (see (A1) and (A2) in the Appendix), we obtain

$$
\begin{gathered}
I_{x_{1}} \equiv \lambda_{1}\left|\frac{\partial D_{\mathrm{n}_{1}}}{\partial x_{1}}\right|=\lambda_{1} \frac{\beta^{2} \tau_{x}}{\gamma \alpha}, \\
I_{x_{2}} \equiv \lambda_{2}\left|\frac{\partial D_{\mathrm{n}_{2}}}{\partial x_{2}}\right|=\lambda_{2} \frac{\alpha^{2} \tau_{x}}{\gamma \beta} .
\end{gathered}
$$

Note that both trading intensities are a function of agents' conjectured values for $\alpha$ and $\beta$, as they use the function in (1) in order to update their beliefs about $\theta$ (which then influences their demand for the risky asset). By rewriting the aggregate demand functions of the rational traders in a general way, we are able to find a connection between the implied values of $\alpha$ and $\beta$ (that follow from invoking rational 
expectations) and the trading intensities:

$$
\begin{aligned}
\int_{0}^{1} D_{\mathrm{f}} \mathrm{df} & =\frac{\tau_{\epsilon}}{\gamma} \theta+\tilde{P}_{\mathrm{f}} P, \\
\int_{0}^{\lambda_{1}} D_{\mathrm{n}_{1}} \mathrm{dn}_{1} & =\tilde{P}_{\mathrm{n}_{1}} P-I_{x_{1}} x_{1}, \\
\int_{0}^{\lambda_{2}} D_{\mathrm{n}_{2}} \mathrm{dn}_{2} & =\tilde{P}_{\mathrm{n}_{2}} P-I_{x_{2}} x_{2}, \\
\int_{0}^{\lambda_{u}} D_{\mathrm{u}} \mathrm{du} & =\tilde{P}_{\mathrm{u}} P,
\end{aligned}
$$

where $\tilde{P}_{\mathrm{f}}, \tilde{P}_{\mathrm{n}_{1}}, \tilde{P}_{\mathrm{n}_{2}}$, and $\tilde{P}_{\mathrm{u}}$ are functions of the exogenous parameters of the model that correspond to (A1) - (A4) in the Appendix. Thus, in general form, the implied values of the three REE coefficients are given by

$$
\begin{aligned}
a_{\theta} & =\frac{\tau_{\epsilon}}{-\gamma\left(\tilde{P}_{\mathrm{f}}+\tilde{P}_{\mathrm{n}_{1}}+\tilde{P}_{\mathrm{n}_{2}}+\tilde{P}_{\mathrm{u}}\right)}, \\
a_{1} & =\frac{1-I_{x_{1}}}{-\left(\tilde{P}_{\mathrm{f}}+\tilde{P}_{\mathrm{n}_{1}}+\tilde{P}_{\mathrm{n}_{2}}+\tilde{P}_{\mathrm{u}}\right)}, \\
a_{2} & =\frac{1-I_{x_{2}}}{-\left(\tilde{P}_{\mathrm{f}}+\tilde{P}_{\mathrm{n}_{1}}+\tilde{P}_{\mathrm{n}_{2}}+\tilde{P}_{\mathrm{u}}\right)} .
\end{aligned}
$$

Hence, the implied value for $\alpha$ can be expressed as

$$
\alpha=\frac{\tau_{\epsilon}}{\gamma\left(1-I_{x_{1}}\right)}, \quad I_{x_{1}} \in[0,1)
$$

Applying the same logic to the case of the implied value for $\beta$ yields

$$
\beta=\frac{\tau_{\epsilon}}{\gamma\left(1-I_{x_{2}}\right)}, \quad I_{x_{2}} \in[0,1)
$$

(9) and (10) show that the trading intensities are positively connected to the values for $\alpha$ and $\beta$. This is intuitive as more aggressive trading against noise makes the price react less strongly to noise relative to fundamentals. Since $\alpha$ and $\beta$ can be seen as proxies for the informativeness of the price about fundamentals, this is equivalent to a rise in their values.

The defined value range of the two trading intensities is deduced from the fact that $\alpha$ and $\beta$ are always positive, whenever an REE exists. Negative values for $\alpha$ and/or $\beta$ would contradict rational traders' initial conjecture of the price function in equilibrium (see (1)). Consequently, the trading intensities are higher in the HIE 
than in the LIE. In other words, more noise is offset in the HIE than in the LIE.

\section{B. Strategic Complementarities in Trading}

As outlined in the Introduction, one central question is how the non-fundamentally informed traders interact in the financial market, i.e., how their trading intensities are connected. Does a higher $I_{x_{1}}$ lead to a higher or lower $I_{x_{2}}$ and vice versa? By plugging (9) and (10) into (7) and (8), respectively, and rearranging terms, we get

$I_{x_{i}}=\frac{\lambda_{i} \tau_{x} \tau_{\epsilon}}{\gamma^{2}\left(1-I_{x_{j}}\right)^{2}+\lambda_{i} \tau_{x} \tau_{\epsilon}}, \quad$ for $i, j=1,2, j \neq i$

The general form of (11) follows from the symmetry of (7) and (8). By inspecting (11), the next proposition immediately follows.

Proposition 2. Trading against $x_{i}$ is a complement to trading against $x_{j}$, i.e., $\partial I_{x_{i}} / \partial I_{x_{j}}>0$.

The explanation for the clear complementarity is the following: A higher $I_{x_{j}}$ means that more noise coming from the $x_{j}$-noise traders is offset. This benefits the traders that do not know $x_{j}$, as they now are able to obtain a more precise signal about $\theta$ from disentangling the market price. As a consequence, they trade more aggressively on their signal for $\theta$ generated out of the market price. Since the $x_{i}$-informed traders exclusively use their non-fundamental information in order to extract noise from the market price, more aggressive trading on their obtained price signal is equivalent to more aggressive trading against $x_{i}$, i.e., a higher $I_{x_{i}}$.

The identified inference augmentation effect exerts the opposite effect compared to GY 2015, who deal with diverse fundamental information. In their model, it favors strategic substitutability in trading. They additionally identify an uncertainty reduction effect, which boosts complementarities in trading. Thus, the resulting type of interaction is ambiguous in their setup. Trading against different types of noise, by contrast, is unambiguously a complement.

\section{An Explanation for Equilibrium Multiplicity}

The clear complementarity in trading against different types of noise is the driving mechanism that leads to equilibrium multiplicity. Noise-informed traders' interaction at the trading stage leads to the existence of two self-fulfilling REE. Since agents' conjecture about the values for $\alpha$ and $\beta$ influences how well the market price reflects the fundamental asset value, $\theta$, it also affects how aggressively agents trade against 
noise. Thus, the conjectured values for $\alpha$ and $\beta$ shape the trading intensities (see (7) and (8)).

If rational agents conjecture, e.g., a high $\alpha$, the market price becomes a precise signal for $\theta$ for the $x_{2}$-informed traders, which makes them trade very aggressively against their non-fundamental information, i.e., $I_{x_{2}}$ rises. By (11), a high $I_{x_{2}}$ leads to a high $I_{x_{1}}$ due to the complementarities in trading. A high $I_{x_{1}}$ unambiguously translates into a high implied value for $\alpha$ (see (9)). Hence, the initial conjecture of the rational agents is verified in equilibrium, thereby leading to the existence of the HIE. The symmetric argument holds for the conjecture and verification of a high value for $\beta$. By contrast, the conjecture and verification of low values for $\alpha$ and for $\beta$ justify the existence of the LIE.

\section{Price Informativeness}

As already outlined, $\alpha$ and $\beta$ can be seen as proxies for the informativeness of the market price about fundamentals. In equilibrium, the information that, e.g., $\alpha$ conveys can be split up into two parts as follows (see (A6) in the Appendix):

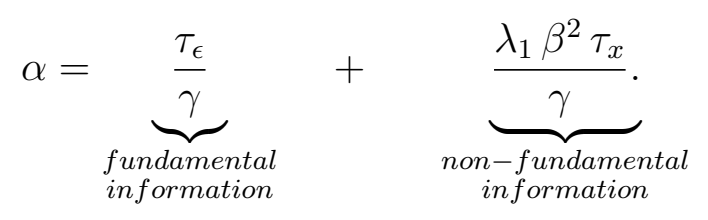

The first component of $\alpha$ determines how much fundamental information it conveys. It is given by the trading intensity of the fundamentally informed traders with respect to their private signal about $\theta$. This component is comparable to the one of the seminal model of GS 1980. The second component of $\alpha$ determines how much information about fundamentals the price conveys due to the existence of non-fundamental information. It also shows the crucial connection between the two REE fractions and, hence, between the two trading intensities. $\alpha$ and $\beta$ are clearly positively connected. Thus, the amount of information $\alpha$ conveys also depends on the amount of information that $\beta$ conveys and vice versa. This positive link between the two REE fractions gives rise to the explained complementarity in trading against different types of noise. As a consequence, the interaction of the noise-informed agents benefits price informativeness. Following Vives (2008), we define this metric as the inverse of the variance of fundamentals conditional on the market price. It is given by

$\frac{1}{\operatorname{Var}[\theta \mid P]}=\tau_{\theta}+\frac{\tau_{x} \tau_{\epsilon}^{2}}{\gamma^{2}\left[\left(1-I_{x_{1}}\right)^{2}+\left(1-I_{x_{2}}\right)^{2}\right]}$. 
Hence, the effect of a rise in $I_{x_{i}}$ on price informativeness is given as follows:

$$
\frac{d \operatorname{Var}^{-1}[\theta \mid P]}{d I_{x_{i}}}=\underbrace{\frac{\partial \operatorname{Var}^{-1}[\theta \mid P]}{\partial I_{x_{i}}}}_{\text {direct ef fect }}+\underbrace{\frac{\partial \operatorname{Var}^{-1}[\theta \mid P]}{\partial I_{x_{j}}} \frac{d I_{x_{j}}}{d I_{x_{i}}}}_{\begin{array}{c}
\text { complementarity } \\
\text { effect }
\end{array}} \text {, for } i, j=1,2, i \neq j
$$

According to (13), the total effect can be split up into two parts. First, by increasing their trading intensity, the $x_{i}$-informed agents counteract more noise induced by the $x_{i}$-noise traders. This raises the quality of the market price as an adequate signal for the fundamental asset value. This fact is represented by the first summand in (13). Additionally, a higher $I_{x_{i}}$ triggers the derived complementarity in trading against noise. A rise in $I_{x_{i}}$ leads to a rise in $I_{x_{j}}$ (see (11)). If more noise generated by the $x_{i}$-noise traders is counteracted, the $x_{j}$-informed traders increase their own trading intensity. The rise in $I_{x_{j}}$ further improves price informativeness as more noise coming from the $x_{j}$-noise traders is offset as a consequence. This connection is described by the second summand in (13). Higher trading intensities increase price informativeness through two channels. Due to this positive relationship, price informativeness is, of course, higher in the HIE than in the LIE.

\section{E. Consequences of a Rise in $\lambda_{i}$ in Equilibrium}

Next, we would like to examine the effects of an increase in the mass of noiseinformed traders in equilibrium. On the one hand, we are interested in the influence on the trading intensities induced by a change in $\lambda_{1}$ or $\lambda_{2}$. By (11), it can be seen that a rise in $\lambda_{i}$ (resp., $\lambda_{j}$ ) directly affects $I_{x_{i}}$ (resp. $I_{x_{j}}$ ). Additionally, a change in $\lambda_{i}$ (resp. $\lambda_{j}$ ) indirectly affects $I_{x_{i}}$ (resp., $I_{x_{j}}$ ) through influencing the respective other trading intensity. A change in $\lambda_{i}$ (resp., $\lambda_{j}$ ) only indirectly affects $I_{x_{j}}$ (resp., $I_{x_{i}}$ ) by changing $I_{x_{i}}$ (resp., $I_{x_{j}}$ ). On the other hand, we explore the effects the mass of noise-informed traders exerts on the existence and multiplicity of equilibria in the model. The results are summarized in the following proposition (with the proof and the expression for $\tilde{\lambda}_{i}$ in (A19) in the Appendix):

\section{Proposition 3.}

(a) The total effect of a rise in $\lambda_{i}$ on both trading intensities is given by

$$
\begin{aligned}
& \frac{d I_{x_{i}}}{d \lambda_{i}}=\Gamma^{-1} \times \frac{\partial I_{x_{i}}}{\partial \lambda_{i}}, \\
& \frac{d I_{x_{j}}}{d \lambda_{i}}=\Gamma^{-1} \times \frac{\partial I_{x_{j}}}{\partial I_{x_{i}}} \frac{\partial I_{x_{i}}}{\partial \lambda_{i}},
\end{aligned}
$$




$$
\Gamma \equiv 1-4 I_{x_{1}} I_{x_{2}}
$$

(b) In the LIE (resp., HIE), it holds that $\Gamma>0$ (resp., $\Gamma<0)$.

(c) If $\lambda_{i}=\tilde{\lambda}_{i}$, it holds that

$$
\begin{aligned}
& \alpha=\frac{2 \lambda_{2} \tau_{\epsilon} \tau_{x}+\sqrt{\lambda_{2} \tau_{\epsilon} \tau_{x}\left(4 \lambda_{2} \tau_{\epsilon} \tau_{x}+3 \gamma^{2}\right)}}{3 \gamma \lambda_{2} \tau_{x}}, \\
& \beta=\frac{2 \lambda_{1} \tau_{\epsilon} \tau_{x}+\sqrt{\lambda_{1} \tau_{\epsilon} \tau_{x}\left(4 \lambda_{1} \tau_{\epsilon} \tau_{x}+3 \gamma^{2}\right)}}{3 \gamma \lambda_{1} \tau_{x}} .
\end{aligned}
$$

(d) If $\lambda_{i}>\tilde{\lambda}_{i}$, there is no equilibrium. If $\lambda_{i}<\tilde{\lambda}_{i}$, there are two equilibria.

Since all partial derivatives in (14) and (15) are clearly positive (see (11)), part (a) in proposition 3 states that a rise in $\lambda_{i}$ can decrease both trading intensities in equilibrium, if $\Gamma<0$. Part (b) shows that this always happens in the HIE, whereas the opposite effect holds in the LIE. That is, the direction of influence on the trading intensities induced by a rise in the mass of noise-informed traders is pinned down by the equilibrium rational traders coordinate on.

The obtained result is in line with the existing literature on non-fundamental information and equilibrium multiplicity. Comparable results are derived in Ganguli \& Yang (2009). In their specification, a rise in the mass of informed agents increases informativeness in one equilibrium while decreasing it in a second one. I identify this feature in my model, too.

The fact that a rise in the mass of noise-informed agents can lead to a decrease in the trading intensities (and equilibrium fractions) stems, as in the case of equilibrium multiplicity, from the self-fulfilling expectations of the rational traders. The reasoning I use is similar to the one in Ganguli \& Yang (2009, p. 100): Suppose the agents coordinate on the HIE and, hence, conjecture that a rise in the mass of noise-informed agents leads to a fall in the equilibrium fractions (and in the trading intensities). The implied values of $\alpha$ and $\beta$ as functions of their conjectured values are given by (A6) and (A7) in the Appendix. According to these two equations, the direct effect of a rise in the mass of non-fundamentally informed traders is positive, thereby tending to increase the implied values of the two fractions. However, since the conjectured values of $\alpha$ and $\beta$ are high in the HIE (in comparison to their values in the LIE), the induced decline in the conjectured values outweighs the direct positive effect. In aggregate, this leads to lower implied values for $\alpha$ and $\beta$, which self-fulfills agents' initial conjecture.

Moreover, part (c) in proposition 3 shows that there exists a critical value for $\lambda_{i}$ 
which leads to a unique REE. In this special case, the solutions for $\alpha$ and $\beta$ can be determined in closed form. They are given by (17) and (18), respectively. According to part (d), there are two equilibria (the LIE and the HIE) if the mass of noiseinformed traders is sufficiently low. If the overall mass of noise-informed traders is too high, an equilibrium fails to exist and the market breaks down. By carefully inspecting $f(\alpha)$ and $f(\beta)$ in proposition 1 , it can be seen that the same occurs for sufficiently high values of $\tau_{\epsilon}$ and $\tau_{x}$ and for sufficiently low values of $\gamma$.

Strong informed trading expressed by a high mass of noise-informed agents (i.e, a high $\lambda_{1}$ or $\lambda_{2}$ ) or a precise fundamental signal (i.e., a high $\tau_{\epsilon}$ ) exacerbates the adverse selection problem in financial markets. Very aggressive trading expressed by low risk aversion (i.e., a low $\gamma$ ) produces the same effect. Adverse selection in financial markets describes the state that traders are exposed to the risk of potentially trading against other agents that possess information superior to their own one (see, e.g., Medrano \& Vives (2004)).

If the adverse selection problem happens to be very intense, agents might refrain from participating in the market, thereby producing a market breakdown. The fact that too much informed trading leads to a market breakdown due to the described adverse selection problem can also be found in other models related to non-fundamental information like in Ganguli \& Yang (2009) and in Marmora \& Rytchkov (2018).

In my setup, the novelty compared to Ganguli \& Yang (2009) and Marmora \& Rytchkov (2018) is that the volatility of noise trading also influences the existence of an equilibrium. This result can be directly linked to the adverse selection problem in financial markets, too. Highly volatile noise trading (i.e., a low $\tau_{x}$ ) alleviates this problem. If the impact of noise traders increases, the risk of trading against a better informed agent is mitigated. Instead, it becomes more likely to trade against an uninformed noise trader (cf. also Vives (2008, Chapter 4)).

\section{Costly Signals and Information Acquisition}

\section{A. Information Acquisition Equilibrium}

In section 2 , knowing $x_{1}$ and $x_{2}$ is not linked to any cost. In what follows, this assumption is relaxed and $x_{1}$ and $x_{2}$ are turned into costly signals. Particularly, information on $x_{1}$ and on $x_{2}$ can be acquired at cost $c_{1}>0$ and at cost $c_{2}>0$, respectively. This allows us to endogenize the values for $\lambda_{1}$ and $\lambda_{2}$. For the sake of tractability, each uninformed trader is only able to acquire one of the two signals.

\footnotetext{
${ }^{6}$ Imposing a restriction on the overall mass of fundamentally uninformed traders would significantly complicate the derivation of an equilibrium in the information market, however leaving all derived results qualitatively unchanged.
} 
Throughout the analysis, we assume, similar to GY 2015, that there are always some rational traders that decide to stay completely uninformed in equilibrium, i.e., $\lambda_{\mathrm{u}}>0$. This allows us to omit the analysis of corner solutions where all fundamentally uninformed agents decide to acquire information on $x_{1}$ and on $x_{2}$, respectively. ${ }^{6}$ As already pointed out by GY (2015, p. 1740), the "case of $\lambda_{u}>0$ is of course empirically relevant, since in reality it is unlikely that every trader is informed." As a consequence, we are interested in the following four outcomes in equilibrium: $\left(\lambda_{1}^{*}=\lambda_{2}^{*}=0\right),\left(\lambda_{1}^{*}>0, \lambda_{2}^{*}=0\right),\left(\lambda_{1}^{*}=0, \lambda_{2}^{*}>0\right)$, and $\left(\lambda_{1}^{*}>0, \lambda_{2}^{*}>0\right)$.

By comparing the ex-ante expected utility of a noise-informed trader with the one of an uninformed trader, we can derive the value of non-fundamental information. It is given in the next proposition (with the proof in the Appendix).

Proposition 4. The value of information about noise is given by

$\phi_{x_{i}}=\frac{1}{2 \gamma} \log \left[\frac{\mathbb{V a r}[\theta \mid P]}{\operatorname{Var}\left[\theta \mid P, x_{i}\right]}\right]$, for $i=1,2$.

According to (19), the value of non-fundamental information is mainly shaped by the ratio of the uncertainty that traders face if they only observe the market price and by the variance if they additionally know $x_{i}$. The more the information about $x_{i}$ reduces the conditional variance of $\theta$ compared to just observing the market price, the higher is its value. If the information about noise only marginally reduces the uncertainty the traders are confronted with, its value is rather small.

In general, a rational agent is willing to acquire information about noise if its cost is lower than its value. If its cost exactly equals its benefit, the agent is indifferent between becoming noise-informed or not. Since we suppose that there are always some rational agents that decide to stay completely uniformed, the cost of a signal must be equal to its value given that there are noise-informed traders in the market. That is, if $\lambda_{i}^{*}>0$, we have $c_{i}=\phi_{x_{i}}$. A trader refrains from acquiring information about $x_{i}$ if the cost is higher than its value. Thus, if $\lambda_{i}^{*}=0$, we have $c_{i} \geq \phi_{x_{i}}$. The following proposition describes the equilibrium in the information market (with the proof and the expressions for $\bar{c}, \bar{c}_{2}, f\left(c_{1}\right)$, and $g\left(c_{1}\right)$ in the Appendix):

\section{Proposition 5.}

(a) $\lambda_{1}^{*}=\lambda_{2}^{*}=0$ is an equilibrium if $c_{1} \geq \bar{c}$ and $c_{2} \geq \bar{c}$.

(b) $\lambda_{1}^{*}>0, \lambda_{2}^{*}=0$ is an equilibrium if $c_{1}<\bar{c}$ and $c_{2} \geq f\left(c_{1}\right)$. 
(c) $\lambda_{1}^{*}=0, \lambda_{2}^{*}>0$ is an equilibrium if $c_{1}>\bar{c}$ and $\bar{c} \geq c_{2} \geq g\left(c_{1}\right)$.

(d) $\lambda_{1}^{*}>0, \lambda_{2}^{*}>0$ is an equilibrium if

(i) $c_{1} \leq \bar{c}$ and $\bar{c}_{2}>c_{2}>f\left(c_{1}\right)$;

(ii) $c_{1}>\bar{c}$ and $\bar{c}_{2}>c_{2}>g\left(c_{1}\right)$.

(e) There is no equilibrium in the information market if

(i) $c_{1} \leq \bar{c}$ and $c_{2}<f\left(c_{1}\right)$;

(ii) $c_{1}>\bar{c}$ and $c_{2}<g\left(c_{1}\right)$.

Building on proposition 5, Figure 2 illustrates all possible equilibria in the in-

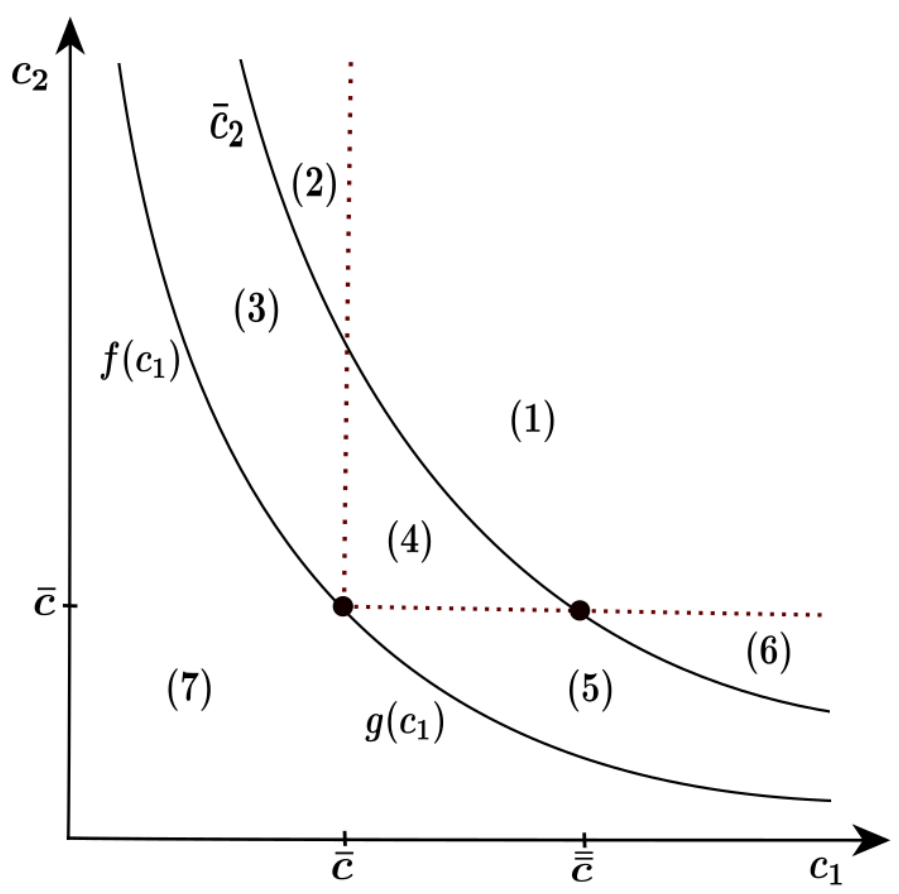

Figure 2: Information Acquistion Equilibrium

formation market in the space of $\left(c_{1}, c_{2}\right)$, where the value for $\overline{\bar{c}}$ directly follows from equating $\bar{c}$ with $\bar{c}_{2}$ and solving for $c_{1}$. In space (1), both cost parameters are too high and all agents refrain from acquiring non-fundamental information (i.e., $\lambda_{1}^{*}=\lambda_{2}^{*}=0$ ). In space (2), agents only acquire information about $x_{1}$ (i.e., $\lambda_{1}^{*}>0, \lambda_{2}^{*}=0$ ). Spaces (3) - (5) define a channel that supports multiple equilibria in the model. In these three spaces an equilibrium with both groups of noise-informed traders (i.e., $\lambda_{1}^{*}>0$, $\left.\lambda_{2}^{*}>0\right)$ is always possible. Additionally, there is a second equilibrium in the information market, whose type depends on the exact combination for $c_{1}$ and $c_{2}$. Space (3) (resp., space (5)) also supports an equilibrium of the form $\lambda_{1}^{*}>0, \lambda_{2}^{*}=0$ (resp., 
$\left.\lambda_{1}^{*}=0, \lambda_{2}^{*}>0\right)$. In space (4), an equilibrium without non-fundamentally informed traders (i.e., $\lambda_{1}^{*}=\lambda_{2}^{*}=0$ ) is possible. In space (6), equilibrium is unique and of the form $\lambda_{1}^{*}=0, \lambda_{2}^{*}>0$.

Surprisingly, an information acquisition equilibrium fails to exist for sufficiently small costs (see space (7)). Intuitively, one would expect an equilibrium with both groups of noise-informed traders in this situation. The explanation for this phenomenon is the following: In a potential equilibrium of the form $\lambda_{1}^{*}>0, \lambda_{2}^{*}>0$, low costs are associated with low values of information about noise. Low values of information about noise are associated with low values for $\alpha$ and $\beta$ in a potential equilibrium. This is intuitive as low values for $\alpha$ and $\beta$ imply a rather uninformative price. Thus, knowing one of the two noise shocks does not significantly improve the predictive power of the market price with respect to the fundamental asset value. It still remains a rather noisy signal for $\theta$. As a consequence, the value of information about noise is low. However, if $\alpha$ and $\beta$ are smaller than $\tau_{\epsilon} / \gamma$, i.e., if they are smaller than the trading intensity of the fundamentally informed agents with respective to their private signal about $\theta$, an information acquisition equilibrium with both groups of non-fundamentally informed agents present can not exist (see also (A6) and (A7) in the Appendix). That is, in order to be able to carry non-fundamental information, both $\alpha$ and $\beta$ need to convey more information than potentially contributed by the fundamentally informed traders. For sufficiently small values of $c_{1}$ and $c_{2}$ and, hence, for sufficiently low values of information about noise, this is not the case and an information acquisition equilibrium with non-fundamentally informed traders fails to exist. $^{7}$

\section{B. Interactions in the Information Market}

After having derived an equilibrium with endogenous values for $\lambda_{1}$ and $\lambda_{2}$, we now turn to the strategic interactions in information acquisition. In other words, we would like analyze whether acquiring information on the same noise component on the one hand and on different noise components on the other hand is a strategic complement and substitute, respectively. If a rise in $\lambda_{\mathrm{i}}$ increases (resp., decreases) $\phi_{x_{i}}$, acquiring information about the same noise component is said to be a complement (resp., substitute). That is, as more traders with information about $x_{i}$ enter the market, the incentive for other agents to acquire information about $x_{i}$ rises (resp., shrinks), which is expressed by a higher (resp., lower) value of information about noise. Whenever a rise in $\lambda_{\mathrm{j}}$ increases (resp., decreases) $\phi_{x_{i}}$, acquiring information

\footnotetext{
${ }^{7}$ The obtained result would not qualitatively change if one allowed for the existence of the excluded corner solutions. There still would exist a space near the origin in Figure 2 where an equilibrium in the information market would fail to exist.
} 
about different noise components is a complement (resp., substitute), i.e., as more traders with information about $x_{j}$ enter the market, the incentive for other traders to acquire information about $x_{i}$ rises (resp., shrinks).

Analogously to GY 2015, (19) can be split up into two parts as follows:

$$
\phi_{x_{i}}=\frac{1}{2 \gamma} \underbrace{\log \left[\tau_{\theta}+\frac{\tau_{\epsilon}^{2} \tau_{x}}{\gamma^{2}\left(1-I_{x_{j}}\right)^{2}}\right]}_{\text {signal precision }}-\frac{1}{2 \gamma} \underbrace{\log \left[\frac{1}{\operatorname{Var}[\theta \mid P]}\right]}_{\text {price informativeness }} .
$$

According to (20), a change in $\lambda_{i}$ or $\lambda_{j}$ affects $\phi_{x_{i}}$ in two ways. On the one hand, it influences the precision of the signal an $x_{i}$-informed trader gains by observing the market price. Since this precision is shaped by how aggressively the $x_{j}$-informed traders trade against their signal about noise, $\lambda_{i}$ and $\lambda_{j}$ affect this component through affecting $I_{x_{j}}$. A(n) decrease (resp., increase) in the named precision reduces (resp., raises) the incentive to acquire information about $x_{i}$. On the other hand, the overall price informativeness is affected by a rise in the mass of noise-informed agents. The more informative the market price, the lower is the incentive to acquire costly non-fundamental information. Whenever an increase in $\lambda_{i}$ or $\lambda_{j}$ raises (resp., decreases) price informativeness, agents' incentive to free-ride on the price increases (resp., shrinks).

In the LIE, a rise in the mass of noise-informed traders increases both trading intensities. Thus, both components in (20) rise and the overall effect on the value of information about noise is ambiguous. The same result holds for the HIE with the respective inverse argumentation. In the HIE, a rise in the mass of non-fundamentally informed agents leads to fall in both trading intensities. Thus, the precision of the price signal an $x_{i}$-informed traders gleans as well as overall price informativeness shrink. The next proposition summarizes the obtained results concerning agents' interactions in the information market (with the proof delegated to the Appendix):

Proposition 6. (a) If $\lambda_{j}=0$, acquiring information about the same noise component is always a substitute, i.e., $d \phi_{x_{i}} / d \lambda_{i}<0$. (b) If $\lambda_{j}>0$, acquiring information about the same noise component can be a complement in the LIE and in the HIE, i.e., $d \phi_{x_{i}} / d \lambda_{i}>0$. (c) Acquiring information about different noise components can be a complement in the LIE and in the HIE, i.e., $d \phi_{x_{i}} / d \lambda_{j}>0$.

The exact conditions that ensure the complementarities mentioned in part (b) and (c) in proposition 6 can also be found in the Appendix. Part (a) in proposition 6 states that a rise in $\lambda_{i}$ always reduces the value of information about $x_{i}$ (i.e., $d \phi_{x_{i}} / d \lambda_{i}<0$ ), if non-fundamental information is not diverse (i.e., $\lambda_{j}=0$ ). In other words, acquiring information about the same noise component is unambiguously a 
substitute whenever there is only one non-fundamentally informed group present. The explanation is the following: If $\lambda_{i}$ rises, $I_{x_{i}}$ increases, too. Since there is no non-fundamental information diversity, there is no equilibrium multiplicity. In this reduced setting, a higher $\lambda_{i}$ always translates into a higher $I_{x_{i}}$. By raising their trading intensity, the $x_{i}$-informed traders turn the market price into a more accurate signal for the risky fundamental asset value. However, since there is no second group of noise-informed traders present, their action induces no complementarity in trading against noise, thereby leaving their residual variance unchanged. As a consequence, their informational advantage shrinks. This reduces the incentive to acquire information about $x_{i}$.

Part (b) in proposition says that acquiring information about the same noise component can be complement in both equilibria if noise is multidimensional and non-fundamental information is diverse. If $\lambda_{j}>0$, a rise in the mass of traders with information about $x_{i}$ does not only affect overall price informativeness, but also the precision of the price signal an $x_{i}$-informed trader obtains through changing $I_{x_{j}}$. In the LIE (resp., in the HIE), the positive affect on the value of information about noise generated by increasing the signal precision (resp., by decreasing overall price informativeness) can outweigh the negative effect generated by increasing overall price informativeness (resp., by decreasing the signal precision). This, then, leads to complementarities in the acquisition of information about the same noise component. Part (c) shows that the same holds true for the acquisition of information about different noise components. Both in the LIE and in the HIE, acquiring information about $x_{i}$ can be a complement to acquiring information about $x_{j}$.

Table 1 compares the obtained results on strategic interactions in acquiring nonfundamental information with the relevant literature that is concerned with multidimensional fundamentals and uniform and diverse fundamental information, respectively - namely GS 1980 and GY 2015. The last two rows in Table 1 display the results contained in proposition 6 . In the seminal GS 1980 model, agents only

Same component Different components

\begin{tabular}{clc}
\hline \hline GS 1980 & substitute & $/$ \\
\hline GY 2015 & substitute & substitute or complement \\
\hline$\lambda_{i}>0, \lambda_{j}=0$ & substitute & $/$ \\
\hline$\lambda_{i}>0, \lambda_{j}>0$ & substitute or complement & substitute or complement \\
\hline
\end{tabular}

Table 1: Comparison of Strategic Interactions in Information Acquistion have information about one of the two risky fundamental components, that jointly determine the fair value of the asset. In their setup, acquiring information about this fundamental is always a substitute. In the extension of GY 2015, with two 
groups of rational traders that possess information about one of the two fundamentals each, it is a substitute, too. Although fundamental information is diverse, acquiring information about the same fundamental is always a substitute.

If noise is multidimensional and non-fundamental information is uniform, acquiring information about the same noise component is always a substitute. This is comparable to the result on fundamental information acquisition obtained by GS 1980. However, if non-fundamental information is diverse, acquiring information about the same noise component can be a complement. A comparable result does not hold when concerning diverse fundamental information, as analyzed by GY 2015 . Nevertheless, GY 2015 uncover a possible complementarity in acquiring information about different fundamental components. When considering multidimensional noise and diverse non-fundamental information, the analogous complementarity can occur, i.e., non-fundamental information acquisition can be characterized by cross-complementarities.

\section{An Extension with Three-Dimensional Noise}

This section briefly reviews the main implications that follow from extending the basic model to the case of three-dimensional noise and adding a third group of noise-informed traders. Introducing a third dimension of noise leads to a highly non-linear system that determines the three REE fractions. As a consequence, the respective fixed-point equations can not be analytically derived anymore. Therefore, the obtained results rely on an extensive set of numerical simulations. ${ }^{8}$

The numerical calibrations show that all of the main results of the model with two-dimensional noise regarding equilibrium multiplicity and complementarities in the financial market and in the information market are still valid. Nevertheless, the three-dimensional extension highlights the importance of diverse non-fundamental information in generating equilibrium multiplicity. If and only if information about all three noise shocks is available to traders, i.e., non-fundamental information is sufficiently diverse, complementarities in trading are strong enough to generate multiple equilibria. As one of the three groups of non-fundamentally informed traders vanishes, equilibrium turns out to be unique.

The most striking difference compared to the two-dimensional case lies in the properties of the equilibria. They significantly differ from the "classical" ones à la Ganguli \& Yang (2009). In both equilibria, a rise in $\lambda_{i}$ now leads to an increase in $I_{x_{i}}$, whereas leading to a fall (resp., a rise) in the other two trading intensities in the HIE (resp., in the LIE). More interestingly, the mass of noise-informed traders does not shape the existence of an equilibrium anymore. If noise is three-dimensional, the

\footnotetext{
${ }^{8}$ The exact numerical simulations are available upon request.
} 
adverse selection problem is less serve than in the two-dimensional case. The higher the dimension of noise, the smaller is the informational advantage obtained from observing a single noise component. ${ }^{9}$ However, if fundamental information is very precise (i.e., a high $\tau_{\epsilon}$ ), noise trading is very weak (i.e., a high $\tau_{x}$ ), or trading is very aggressive (i.e., a low $\gamma$ ), adverse selection intensifies and the market breaks down.

Though much less tractable, extending the basic model to the case with threedimensional noise underscores the importance of non-fundamental information diversity in generating multiple equilibria. Additionally, new properties of equilibria linked to the interactions of noise-informed agents are uncovered, that have been absent in the literature so far. ${ }^{10}$

\section{Conclusion}

This paper explores the strategic interactions between different rational traders that are informed about different components of noise trader demand. It contributes to the existing theoretical literature on non-fundamental information by identifying new types of complementarities in the financial market and in the information market, which can only be uncovered if noise is multidimensional. In the financial market, agents' trades against different order flows from noise traders are complements due to an inference augmentation effect. As one noise-informed group trades more aggressively against the piece of noise trader demand that they observe, the price becomes more informative about fundamentals for the other noise-informed group. This makes them trade more aggressively on their price signal, which implies more aggressive trading against the type of noise they observe. The strong complementarity in trading leads to the existence of multiple, self-fulfilling equilibria, that show, if noise is two-dimensional, similar properties to those of Ganguli \& Yang (2009). If noise is three-dimensional, the properties significantly differ due to weaker adverse selection. Moreover, the complementarity in trading against noise clearly benefits price informativeness.

In the information market, multiple equilibria and complementarities are also possible. I identify a channel for combinations of cost parameters where an equilibrium with diverse non-fundamental information and one with uniform non-fundamental information and without non-fundamental information, respectively, are plausible. If noise is multidimensional and non-fundamental information is diverse, acquiring

\footnotetext{
${ }^{9}$ If one allowed for a group of traders that would observe two of the three noise shocks, the two equilibria again would show the classical properties and a high mass of noise-informed traders would lead to a market breakdown.

${ }^{10}$ The author is aware of the fact that the adverse selection problem vanishes if the error terms in the traders' private fundamental signals are correlated, as shown by Manzano \& Vives (2011). However, the extension of this section shows that adverse selection can be significantly weakened if noise is three-dimensional and non-fundamental information is sufficiently dispersed.
} 
information about the same type of noise can be a complement. There can also exist cross-complementarities, i.e., acquiring information about different types of noise can be a complement.

\section{Appendix}

Proof of Proposition 1. By (2) and by computing the first two conditional moments of $\theta$ via the projection theorem, the demand function of an $x_{1}$-informed trader and of an $x_{2}$-informed trader becomes

$\mathrm{D}_{\mathrm{n}_{1}}=\frac{\beta^{2} \tau_{x}\left(\frac{P}{a_{\theta}}-\frac{1}{\alpha} x_{1}\right)-P\left(\tau_{\theta}+\beta^{2} \tau_{x}\right)}{\gamma}$

and

$\mathrm{D}_{\mathrm{n}_{2}}=\frac{\alpha^{2} \tau_{x}\left(\frac{P}{a_{\theta}}-\frac{1}{\beta} x_{2}\right)-P\left(\tau_{\theta}+\alpha^{2} \tau_{x}\right)}{\gamma}$,

respectively. Analogously, the demand of a fundamentally informed trader and of an uninformed one is

$\mathrm{D}_{\mathrm{f}}=\frac{\tau_{\epsilon} s_{\mathrm{f}}+\frac{\tau_{x}}{\frac{1}{\alpha^{2}}+\frac{1}{\beta^{2}} \frac{P}{a_{\theta}}-P\left(\tau_{\theta}+\tau_{\epsilon}+\frac{\tau_{x}}{\frac{1}{\alpha^{2}}+\frac{1}{\beta^{2}}}\right)}}{\gamma}$.

and

$\mathrm{D}_{\mathrm{u}}=\frac{\frac{\tau_{x}}{\frac{1}{\alpha^{2}}+\frac{1}{\beta^{2}}} \frac{P}{a_{\theta}}-P\left(\tau_{\theta}+\frac{\tau_{x}}{\frac{1}{\alpha^{2}}+\frac{1}{\beta^{2}}}\right)}{\gamma}$,

respectively. By plugging (A1) - (A4) into (6) and rearranging terms, we obtain ${ }^{11}$

$\left(1+\lambda_{u}\right)\left[\tau_{\theta}+\frac{\tau_{x}\left(1-a_{\theta}^{-1}\right)}{\frac{1}{\alpha^{2}}+\frac{1}{\beta^{2}}}\right] P+\lambda_{1}\left[\left(1-a_{\theta}^{-1}\right) \beta^{2} \tau_{x}+\tau_{\theta}\right] P$
$+\lambda_{2}\left[\left(1-a_{\theta}^{-1}\right) \alpha^{2} \tau_{x}+\tau_{\theta}\right] P=\tau_{\epsilon} \theta+\left(\gamma-\frac{\lambda_{1} \beta^{2} \tau_{x}}{\alpha}\right) x_{1}+\left(\gamma-\frac{\lambda_{2} \alpha^{2} \tau_{x}}{\beta}\right) x_{2}$.

\footnotetext{
${ }^{11}$ By the law of large numbers, we get $\int_{0}^{1} s_{\mathrm{f}}=\theta$.
} 
By comparing (1) with (A5) and after some tedious calculations, we obtain

$a_{\theta}=\frac{\alpha^{4} \lambda_{2} \tau_{x}+\beta^{2}\left(\tau_{\epsilon}+\lambda_{1} \beta^{2} \tau_{x}\right)+\alpha^{2}\left(\tau_{\epsilon}+\beta^{2} \tau_{x} \omega\right)}{\alpha^{4} \lambda_{2} \tau_{x}+\beta^{2}\left(\tau_{\epsilon}++\tau_{\theta} \omega+\lambda_{1} \beta^{2} \tau_{x}\right)+\alpha^{2}\left[\tau_{\epsilon}+\left(\beta^{2} \tau_{x}+\tau_{\theta}\right) \omega\right]}$,

where $\omega \equiv 1+\lambda_{1}+\lambda_{2}+\lambda_{u}$.

By the definitions of $\alpha$ and $\beta$, it immediately follows that

$a_{1}=\frac{a_{\theta}}{\alpha} \quad$ and $\quad a_{2}=\frac{a_{\theta}}{\beta}$.

Moreover, invoking rational expectations implies that

$\alpha=\frac{\tau_{\epsilon}}{\gamma-\frac{\lambda_{1} \beta^{2} \tau_{x}}{\alpha}}$

and

$\beta=\frac{\tau_{\epsilon}}{\gamma-\frac{\lambda_{2} \alpha^{2} \tau_{x}}{\beta}}$

Thus,

$\alpha=\frac{\tau_{\epsilon}+\lambda_{1} \beta^{2} \tau_{x}}{\gamma}$

and

$\beta=\frac{\tau_{\epsilon}+\lambda_{2} \alpha^{2} \tau_{x}}{\gamma}$

By simultaneously solving (A6) and (A7) for $\alpha$ and $\beta$, we can find the two fixed-point equations that determine the solutions for both defined ratios:

$\alpha=\frac{\lambda_{1} \lambda_{2}^{2} \alpha^{4} \tau_{x}^{3}+2 \lambda_{1} \lambda_{2} \alpha^{2} \tau_{x}^{2} \tau_{\epsilon}+\lambda_{1} \tau_{x} \tau_{\epsilon}^{2}+\tau_{\epsilon} \gamma^{2}}{\gamma^{3}}$

and

$\beta=\frac{\lambda_{1}^{2} \lambda_{2} \beta^{4} \tau_{x}^{3}+2 \lambda_{1} \lambda_{2} \beta^{2} \tau_{x}^{2} \tau_{\epsilon}+\lambda_{2} \tau_{x} \tau_{\epsilon}^{2}+\tau_{\epsilon} \gamma^{2}}{\gamma^{3}}$.

(A8) and (A9) can be used to find the explicit solutions for both fractions. In what follows, we focus on (A9). Due to symmetry, the analogous results hold for (A8). 
Rearranging (A9) delivers

$\lambda_{1}^{2} \lambda_{2} \tau_{x}^{3} \beta^{4}+2 \lambda_{1} \lambda_{2} \tau_{x}^{2} \tau_{\epsilon} \beta^{2}-\gamma^{3} \beta+\lambda_{2} \tau_{x} \tau_{\epsilon}^{2}+\tau_{\epsilon} \gamma^{2}=0$

The solutions for $\beta$ are obtained by determining the roots of the quartic in (A10). Descartes' rule of signs immediately tells us that there exist either two or zero positive real roots. Hence, the existence of an REE can be ensured if and only if the solution of the quartic in (A10) delivers two positive real roots. Following Dickson (1914, Chapter 4$)$, this is the case whenever the discriminant $\left(\Delta_{\beta}\right)$ of (A10) is non-positive. If it is negative, we have two distinct real roots. A discriminant equal to zero means that there are two identical roots. Then, by the using the formula for the discriminant of a quartic (see, e.g., Dickson (1914, p. 41)), we get

$$
\begin{aligned}
\Delta_{\beta}= & \frac{256}{27} \lambda_{1}^{6} \lambda_{2}^{3} \tau_{x}^{9} \tau_{\epsilon}^{3}\left(4 \lambda_{2} \tau_{x} \tau_{\epsilon}+3 \gamma^{2}\right)^{3} \\
& -\frac{1}{27} \lambda_{1}^{4} \lambda_{2}^{2} \tau_{x}^{6}\left(128 \lambda_{1} \lambda_{2}^{2} \tau_{x}^{3} \tau_{\epsilon}^{3}+144 \lambda_{1} \lambda_{2} \tau_{x}^{2} \tau_{\epsilon}^{2} \gamma^{2}-27 \gamma^{6}\right)^{2}
\end{aligned}
$$

Thus, whenever $\Delta_{\beta}<0$ (resp., $\Delta_{\beta}=0$ ), there exist(s) two (resp., one) REE.

Proof of Proposition 3. Formally, the total effect of a rise in $\lambda_{i}$ on $I_{x_{i}}$ and $I_{x_{j}}$ is given by

$\frac{d I_{x_{i}}}{d \lambda_{i}}=\frac{\partial I_{x_{i}}}{\partial \lambda_{i}}+\frac{\partial I_{x_{i}}}{\partial I_{x_{j}}} \frac{d I_{x_{j}}}{d \lambda_{i}} \quad$ and $\quad \frac{d I_{x_{j}}}{d \lambda_{i}}=\frac{\partial I_{x_{j}}}{\partial I_{x_{i}}} \frac{d I_{x_{i}}}{d \lambda_{i}}$

Thus,

$\frac{d I_{x_{i}}}{d \lambda_{i}}=\frac{\frac{\partial I_{x_{i}}}{\partial \lambda_{i}}}{1-\frac{\partial I_{x_{i}}}{\partial I_{x_{j}}} \frac{\partial I_{x_{j}}}{\partial I_{x_{i}}}}=\left(1-\frac{\partial I_{x_{1}}}{\partial I_{x_{2}}} \frac{\partial I_{x_{2}}}{\partial I_{x_{1}}}\right)^{-1} \frac{\partial I_{x_{i}}}{\partial \lambda_{i}}$

and

$\frac{d I_{x_{j}}}{d \lambda_{i}}=\frac{\frac{\partial I_{x_{j}}}{\partial I_{x_{i}}} \frac{\partial I_{x_{i}}}{\partial \lambda_{i}}}{1-\frac{\partial I_{x_{i}}}{\partial I_{x_{j}}} \frac{\partial I_{x_{j}}}{\partial I_{x_{i}}}}=\left(1-\frac{\partial I_{x_{1}}}{\partial I_{x_{2}}} \frac{\partial I_{x_{2}}}{\partial I_{x_{1}}}\right)^{-1} \frac{\partial I_{x_{j}}}{\partial I_{x_{i}}} \frac{\partial I_{x_{i}}}{\partial \lambda_{i}}$

By (11), we have

$\frac{\partial I_{x_{i}}}{\partial I_{x_{j}}}=\frac{2 \gamma^{2}\left(1-I_{x_{j}}\right) \lambda_{i} \tau_{x} \tau_{\epsilon}}{\left(\gamma^{2}\left(1-I_{x_{j}}\right)^{2}+\lambda_{i} \tau_{x} \tau_{\epsilon}\right)^{2}}, \quad$ for $\quad i, j=1,2, i \neq j$ 
Next, we would like to eliminate $\lambda_{i}$ in (A13). Solving (11) for $\lambda_{i}$ yields

$\lambda_{i}=\frac{\gamma^{2} I_{x_{i}}\left(1-I_{x_{j}}\right)^{2}}{\left(1-I_{x_{i}}\right) \tau_{x} \tau_{\epsilon}}$, for $i, j=1,2, i \neq j$

By plugging (A14) into (A13), we obtain

$\frac{\partial I_{x_{i}}}{\partial I_{x_{j}}}=\frac{2 I_{x_{i}}\left(1-I_{x_{i}}\right)}{1-I_{x_{j}}}$, for $i, j=1,2, i \neq j$.

This gives

$\Gamma \equiv 1-\frac{\partial I_{x_{1}}}{\partial I_{x_{2}}} \frac{\partial I_{x_{2}}}{\partial I_{x_{1}}}=1-4 I_{x_{1}} I_{x_{2}}$

which together with (A12) and (A13) yields (14) to (16). Then, we can also show that

$\frac{d I_{x_{i}}}{d \lambda_{i}}=\frac{\tau_{\epsilon} \tau_{x}\left(1-I_{x_{i}}\right)^{2}}{\gamma^{2}\left(1-I_{x_{j}}\right)^{2}\left(1-4 I_{x_{1}} I_{x_{2}}\right)}$,

$\frac{d I_{x_{j}}}{d \lambda_{i}}=\frac{2 \tau_{\epsilon} \tau_{x}\left(1-I_{x_{i}}\right) I_{x_{j}}}{\gamma^{2}\left(1-I_{x_{j}}\right)\left(1-4 I_{x_{1}} I_{x_{2}}\right)}$.

Next, we assume $I_{x_{1}}=0.25 I_{x_{2}}^{-1}$ so that $\Gamma=0$ and examine the consequences in equilibrium. At this point, the effect of a change in $\lambda_{i}$ on both trading intensities is undefined. Then, by (9) and (10), the value for $\alpha$ in equilibrium in terms of $\beta$ is

$\alpha=\frac{4 \tau_{\epsilon}\left(\gamma \beta-\tau_{\epsilon}\right)}{\gamma\left(3 \gamma \beta-4 \tau_{\epsilon}\right)}$

Equating (A17) with (A6) and rearranging terms delivers the following quadratic function in $\beta$ :

$3 \gamma \lambda_{1} \tau_{x} \beta^{2}-4 \lambda_{1} \tau_{\epsilon} \tau_{x} \beta-\gamma \tau_{\epsilon}=0$

Solving (A18) immediately leads to (18). Due to symmetry, this immediately yields (17). By equating (18) with (A7) and (17) with (A6) and solving for $\lambda_{2}$ and $\lambda_{1}$, respectively, we can derive the critical values for the two parameters that are linked to the existence of a unique REE. After some tedious calculations, we can show that the critical value in general form is given by

$$
\tilde{\lambda}_{i}=\frac{27 \gamma^{6}\left[\sqrt{\lambda_{j} \tau_{\epsilon} \tau_{x}\left(4 \lambda_{j} \tau_{\epsilon} \tau_{x}+3 \gamma^{2}\right)}-\lambda_{j} \tau_{\epsilon} \tau_{x}\right]}{16 \lambda_{j} \tau_{\epsilon}^{2} \tau_{x}^{2}\left[3 \gamma^{2}+2 \lambda_{j} \tau_{\epsilon} \tau_{x}+\sqrt{\lambda_{j} \tau_{\epsilon} \tau_{x}\left(4 \lambda_{j} \tau_{\epsilon} \tau_{x}+3 \gamma^{2}\right)}\right]}>0, \quad \text { for } \quad i, j=1,2, i \neq j .
$$


Since both $f(\alpha)$ and $f(\beta)$ from proposition 1 are increasing in $\lambda_{1}$ and $\lambda_{2}$, we can further conclude that $\lambda_{i}<\tilde{\lambda}_{i}$ is a necessary and sufficient condition for the existence of the LIE and of the HIE. If $\lambda_{i}>\tilde{\lambda}_{i}$, there is no solution to the two fixed-point equations in proposition 1 and an REE fails to exist.

To prove part (b) in proposition 3, it suffices to explore the effect of an increase in $\lambda_{i}$ on the REE fractions $\alpha$ and $\beta$, since they are positively connected to the trading intensities. According to proposition 1, in equilibrium, it holds that $\beta-f(\beta)=0$ (and $\alpha-f(\alpha)=0$ ). Applying the implicit function theorem delivers

$\frac{d \beta}{d \lambda_{i}}=\frac{\frac{d f(\beta)}{d \lambda_{i}}}{1-f^{\prime}(\beta)}$

Since $f(\beta)$ is strictly increasing in $\lambda_{i}$, we have $\operatorname{sign}\left(d \beta / d \lambda_{i}\right)=\operatorname{sign}\left(1-f^{\prime}(\beta)\right)$. As $f(\beta)$ is a strictly increasing and convex function in $\beta$ with a positive intercept, it can be concluded that $f^{\prime}\left(\beta_{L I E}\right)<1$ and $f^{\prime}\left(\beta_{H I E}\right)>1$. This means that $d \beta / d \lambda_{i}>0$ in the LIE and $d \beta / d \lambda_{i}<0$ in the HIE. By symmetry, the analogous result holds for $\alpha_{L I E}$ and $\alpha_{H I E}$. Due to the positive link between the REE fractions and the trading intensities, this proves that $\Gamma>0$ (resp., $\Gamma<0$ ) is true in the LIE (resp., HIE).

Proof of Proposition 4. By turning $x_{1}$ and $x_{2}$ into costly signals, the wealth function of a noise-informed trader slightly changes to $\pi_{\mathrm{n}_{\mathrm{i}}}=(\theta-P) D_{\mathrm{n}_{\mathrm{i}}}-c_{i}$ for $i=1,2$. Due to the CARA form of utility, initial wealth does not affect the investment decision, i.e., $c_{i}$ does not change $D_{\mathrm{n}_{\mathrm{i}}}$. Thus, the conditional expected utility of a noise-informed agent can be written as

$\mathbb{E}\left[U\left(\pi_{\mathrm{n}_{\mathrm{i}}}\right) \mid x_{i}, P\right]=-\exp \left\{\gamma c_{i}\right\} \exp \left\{-\frac{\left(\mathbb{E}\left[\theta \mid x_{i}, P\right]-P\right)^{2}}{2 \mathbb{V} \operatorname{Var}\left[\theta \mid x_{i}, P\right]}\right\}$.

Taking expectations conditional on $P$ yields

$\mathbb{E}\left[U\left(\pi_{\mathrm{n}_{\mathrm{i}}}\right) \mid P\right]-\exp \left\{\gamma c_{i}\right\} \mathbb{E}\left[\exp \left\{-\frac{\left(\mathbb{E}\left[\theta \mid x_{i}, P\right]-P\right)^{2}}{2 \mathbb{V} \operatorname{ar}\left[\theta \mid x_{i}, P\right]}\right\} \mid P\right]$

Let $\mathrm{Y}$ be a normally distributed variable. It is well known that the following holds (see, e.g., Demange \& Laroque (1995)):

$\mathbb{E}\left[-y^{2}\right]=\frac{1}{\sqrt{1+2 \mathbb{V a r}[Y]}} \exp \left\{-\frac{(\mathbb{E}[Y])^{2}}{1+2 \mathbb{V a r}[Y]}\right\}$

Applying (A21) with $Y=\left(\mathbb{E}\left[\theta \mid x_{i}, P\right]-P\right) / \sqrt{2 \mathbb{V a r}\left[\theta \mid x_{i}, P\right]}$ and recalling that, by the law of total conditional variance, $\operatorname{Var}\left[\mathbb{E}\left[\theta \mid x_{i}, P\right] \mid P\right]=\mathbb{V} a r[\theta \mid P]-\operatorname{Var}\left[\theta \mid x_{i}, P\right]$, 
conditional on $P$, (A20) becomes

$\mathbb{E}\left[U\left(\pi_{\mathrm{n}_{\mathrm{i}}}\right) \mid P\right]=-\exp \left\{\gamma c_{i}\right\} \sqrt{\frac{\operatorname{Var}\left[\theta \mid x_{i}, P\right]}{\operatorname{Var}[\theta \mid P]}} \exp \left\{-\frac{(\mathbb{E}[\theta \mid P]-P)^{2}}{2 \mathbb{V a r}[\theta \mid P]}\right\}$.

Again taking (unconditional) expectations yields

$\mathbb{E}\left[U\left(\pi_{\mathrm{n}_{\mathrm{i}}}\right)\right]=-\exp \left\{\gamma c_{i}\right\} \sqrt{\frac{\operatorname{Var}\left[\theta \mid x_{i}, P\right]}{\operatorname{Var}[\theta \mid P]}} \mathbb{E}\left[\exp \left\{-\frac{(\mathbb{E}[\theta \mid P]-P)^{2}}{2 \mathbb{V a r}[\theta \mid P]}\right\}\right]$

By a similar argument, the ex-ante expected utility of an uninformed trader can be expressed as

$\mathbb{E}\left[U\left(\pi_{u}\right)\right]=-\mathbb{E}\left[\exp \left\{-\frac{(\mathbb{E}[\theta \mid P]-P)^{2}}{2 \mathbb{V a r}[\theta \mid P]}\right\}\right]$

Equating (A22) with (A23) and solving for $c_{i}$ yields (19).

Proof of Proposition 5. Straightforward computations show that $\phi_{x_{1}}$ and $\phi_{x_{2}}$ can be expressed in terms of $\alpha$ and $\beta$ as follows:

$\phi_{x_{1}}(\alpha, \beta)=\frac{1}{2 \gamma} \log \left[\frac{\left(\alpha^{2}+\beta^{2}\right)\left(\tau_{\theta}+\beta^{2} \tau_{x}\right)}{\beta^{2} \tau_{\theta}+\alpha^{2}\left(\tau_{\theta}+\beta^{2} \tau_{x}\right)}\right]$

$\phi_{x_{2}}(\alpha, \beta)=\frac{1}{2 \gamma} \log \left[\frac{\left(\alpha^{2}+\beta^{2}\right)\left(\tau_{\theta}+\alpha^{2} \tau_{x}\right)}{\beta^{2} \tau_{\theta}+\alpha^{2}\left(\tau_{\theta}+\beta^{2} \tau_{x}\right)}\right]$.

Case 1. First, we look at the case where no one acquires information about noise, i.e., $\lambda_{1}^{*}=\lambda_{2}^{*}=0$. By (A6) and (A7), this leads to $\alpha=\beta=\tau_{\epsilon} / \gamma$. In this situation, no agent finds it beneficial to acquire information about noise given that there is no single trader in the market possessing non-fundamental information. Hence, in equilibrium, it follows that $c_{1} \geq \phi_{x_{1}}\left(\tau_{\epsilon} / \gamma, \tau_{\epsilon} / \gamma\right)$ and $c_{2} \geq \phi_{x_{2}}\left(\tau_{\epsilon} / \gamma, \tau_{\epsilon} / \gamma\right)$. The respective computations yield due to the symmetry of (A24) and (A25) that

$\phi_{x_{1}}\left(\tau_{\epsilon} / \gamma, \tau_{\epsilon} / \gamma\right)=\phi_{x_{2}}\left(\tau_{\epsilon} / \gamma, \tau_{\epsilon} / \gamma\right)=\frac{1}{2 \gamma} \log \left[\frac{2\left(\gamma^{2} \tau_{\theta}+\tau_{\epsilon}^{2} \tau_{x}\right)}{2 \gamma^{2} \tau_{\theta}+\tau_{\epsilon}^{2} \tau_{x}}\right] \equiv \bar{c}$

Therefore, in an information acquisition equilibrium of the form $\lambda_{1}^{*}=\lambda_{2}^{*}=0$, it holds that

$$
c_{1} \geq \bar{c} \quad \text { and } \quad c_{2} \geq \bar{c} .
$$


Case 2. In the second case, we turn to the situation where agents acquire information about $x_{1}$ only, i.e., $\lambda_{1}^{*}>0, \lambda_{2}^{*}=0$. Since $\lambda_{2}^{*}=0$, it follows by (A7) that $\beta=\tau_{\epsilon} / \gamma$. Thus, in equilibrium, we have

$$
\phi_{x_{1}}\left(\alpha, \tau_{\epsilon} / \gamma\right)=c_{1} \quad \text { and } \quad \phi_{x_{2}}\left(\alpha, \tau_{\epsilon} / \gamma\right) \leq c_{2}
$$

The value of $\alpha$ in terms of $c_{1}$ is then given by

$\alpha=\frac{\tau_{\epsilon} \sqrt{\tau_{\epsilon}^{2} \tau_{x}-\left(e^{2 \gamma c_{1}}-1\right) \gamma^{2} \tau_{\theta}}}{\gamma \sqrt{\left(e^{2 \gamma c_{1}}-1\right)\left(\tau_{\epsilon}^{2} \tau_{x}+\gamma^{2} \tau_{\theta}\right)}}$.

From (A6), we know that $\lambda_{1}^{*}$ can be expressed as

$\lambda_{1}^{*}=\frac{\gamma \alpha-\tau_{\epsilon}}{\beta^{2} \tau_{x}}$

Thus, by (A28), for $\lambda_{1}^{*}>0$ to be true in equilibrium, it must hold that $\alpha>\tau_{\epsilon} / \gamma$. By recalling (A27), it can be shown that $\alpha>\tau_{\epsilon} / \gamma$ is equal to

$c_{1}<\frac{1}{2 \gamma} \log \left[\frac{2\left(\gamma^{2} \tau_{\theta}+\tau_{\epsilon}^{2} \tau_{x}\right)}{2 \gamma^{2} \tau_{\theta}+\tau_{\epsilon}^{2} \tau_{x}}\right]=\bar{c}$

Furthermore, by (A27), we can express the value of information about $x_{2}$ as

$\phi_{x_{2}}\left(\alpha, \tau_{\epsilon} / \gamma\right)=\frac{1}{2 \gamma} \log \left[\frac{e^{2 \gamma c_{1}}\left[\left(e^{2 \gamma c_{1}}-1\right) \gamma^{4} \tau_{\theta}^{2}+\tau_{\epsilon}^{4} \tau_{x}^{2}\right]}{\left(e^{2 \gamma c_{1}}-1\right)\left(\gamma^{2} \tau_{\theta}+\tau_{\epsilon}^{2} \tau_{x}\right)^{2}}\right] \equiv f\left(c_{1}\right)$

Therefore, in order for an equilibrium of the form $\lambda_{1}^{*}>0$ and $\lambda_{2}^{*}=0$ to exist, it must hold that

$$
c_{1} \in(0, \bar{c}) \quad \text { and } \quad c_{2} \geq f\left(c_{1}\right) .
$$

Case 3. The third case deals with the situation where no one possesses information about $x_{1}$ and some agents acquire information about $x_{2}$, i.e., $\lambda_{1}^{*}=0, \lambda_{2}^{*}>0$. This case is symmetric to the second one. Hence, it can be concluded without any further calculations that such an equilibrium satisfies

$$
c_{1} \geq f\left(c_{2}\right) \quad \text { and } \quad c_{2} \in(0, \bar{c})
$$

where

$f\left(c_{2}\right) \equiv \frac{1}{2 \gamma} \log \left[\frac{e^{2 \gamma c_{2}}\left[\left(e^{2 \gamma c_{2}}-1\right) \gamma^{4} \tau_{\theta}^{2}+\tau_{\epsilon}^{4} \tau_{x}^{2}\right]}{\left(e^{2 \gamma c_{2}}-1\right)\left(\gamma^{2} \tau_{\theta}+\tau_{\epsilon}^{2} \tau_{x}\right)^{2}}\right]$. 
Next, we would like to express the value range of $c_{2}$ in (A30) in terms of $c_{1}$. To get there, we first analyze the monotonicity of $f\left(c_{2}\right)$ :

$f^{\prime}\left(c_{2}\right)=\frac{\left(e^{2 \gamma c_{2}}-1\right)^{2} \gamma^{4} \tau_{\theta}^{2}-\tau_{\epsilon}^{4} \tau_{x}^{2}}{\left(e^{2 \gamma c_{2}}-1\right)\left[\left(e^{2 \gamma c_{2}}-1\right) \gamma^{4} \tau_{\theta}^{2}+\tau_{\epsilon}^{4} \tau_{x}^{2}\right]}$.

Hence,

$f^{\prime}\left(c_{2}\right) \gtreqless 0 \Leftrightarrow c_{1} \gtreqless \frac{1}{2 \gamma} \log \left[1+\frac{\tau_{\epsilon}^{2} \tau_{x}}{\gamma^{2} \tau_{\theta}}\right] \equiv \tilde{c}>\bar{c}$.

Consequently, $\tilde{c}$ represents the global minimum of $f\left(c_{2}\right)$ (and $f\left(c_{1}\right)$ ). Moreover, since $f\left(c_{2}\right)$ is a quadratic function of $c_{2}$, the respective equation $c_{1}=f\left(c_{2}\right)$ possesses two solutions for $c_{2}$, i.e., each value of $c_{1} \in \mathbb{R}_{+}$are assigned two values of $c_{2}$. The first one, call it $g\left(c_{1}\right)$, is characterized by $g\left(c_{1}\right)<\tilde{c}$, the second one, call it $h\left(c_{1}\right)$, is characterized by $h\left(c_{1}\right)>\tilde{c}$.

Define $C \equiv \gamma^{2} \tau_{\theta}+\tau_{\epsilon}^{2} \tau_{x}$. Then, direct computations yield

$g\left(c_{1}\right)=\frac{-\tau_{\epsilon}^{4} \tau_{x}^{2}+\gamma^{4} \tau_{\theta}+C^{2} e^{2 \gamma c_{1}}-\sqrt{\left(\tau_{\epsilon}^{4} \tau_{x}^{2}-\gamma^{4} \tau_{\theta}-C^{2} e^{2 \gamma c_{1}}\right)^{2}-4 \gamma^{4} \tau_{\theta}^{2} C^{2} e^{2 \gamma c_{1}}}}{2 \gamma^{4} \tau_{\theta}^{2}}$

and

$h\left(c_{1}\right)=\frac{-\tau_{\epsilon}^{4} \tau_{x}^{2}+\gamma^{4} \tau_{\theta}+C^{2} e^{2 \gamma c_{1}}+\sqrt{\left(\tau_{\epsilon}^{4} \tau_{x}^{2}-\gamma^{4} \tau_{\theta}-C^{2} e^{2 \gamma c_{1}}\right)^{2}-4 \gamma^{4} \tau_{\theta}^{2} C^{2} e^{2 \gamma c_{1}}}}{2 \gamma^{4} \tau_{\theta}^{2}}$.

Since $f\left(c_{2}\right)$ is decreasing in $c_{2}$ for $c_{2}<\tilde{c}, g\left(c_{1}\right)$ is decreasing in $c_{1}$ for $c_{1} \in \mathbb{R}_{+}$. Analogously, as $f\left(c_{2}\right)$ is increasing in $c_{2}$ for $c_{2}>\tilde{c}, h\left(c_{1}\right)$ is increasing in $c_{1}$ for $c_{1} \in \mathbb{R}_{+}$. Thus, $c_{1} \geq f\left(c_{2}\right)$ is equivalent to $h\left(c_{1}\right) \geq c_{2} \geq g\left(c_{1}\right)$.

Recall from (A30) that an equilibrium of the form $\lambda_{1}^{*}=0, \lambda_{2}^{*}>0$ requires $c_{2}<\bar{c}$. Since $h\left(c_{1}\right)>\tilde{c}>\bar{c}$, the value range of $c_{2}$ in the third case in terms of $c_{1}$ is given by $\bar{c}>c_{2} \geq g\left(c_{1}\right)$.

Furthermore, since $\alpha\left(c_{1}=\bar{c}\right)=\tau_{\epsilon} / \gamma($ cf. (A27)), it follows by symmetry that $\beta\left(c_{2}=\bar{c}\right)=\tau_{\epsilon} / \gamma$. As $\phi_{x_{1}}\left(\tau_{\epsilon} / \gamma, \beta\right)\left(\equiv f\left(c_{2}\right)\right)$ is decreasing in $c_{2}$ for $c_{2}<\tilde{c}$ and $\phi_{x_{1}}\left(\tau_{\epsilon} / \gamma, \tau_{\epsilon} / \gamma\right)=\bar{c}, c_{1}$ reaches its infimum at $\bar{c}$ in the third case.

Therefore, the condition in (A30) can also be written as

$$
c_{1}>\bar{c} \quad \text { and } \quad \bar{c}>c_{2} \geq g\left(c_{1}\right) .
$$

Note that $g(\bar{c})=\bar{c}$. Since $g\left(c_{1}\right)$ is decreasing in $c_{1}$, we can conclude that $\bar{c}>g\left(c_{1}\right)$ holds for sure for all $c_{1}>\bar{c}$. 
Case 4. The fourth and last case implies that both information about $x_{1}$ and $x_{2}$ is acquired in equilibrium, i.e., $\lambda_{1}^{*}>0, \lambda_{2}^{*}>0$. Thus, it holds that

$$
\phi_{x_{1}}(\alpha, \beta)=c_{1} \quad \text { and } \quad \phi_{x_{2}}(\alpha, \beta)=c_{2} .
$$

First, we derive how a change in $c_{1}$ affects the equilibrium values of $\alpha$ and $\beta$. Implicit differentiation of the system in (A32) with respect to $c_{1}$ yields

$$
\left\{\begin{aligned}
\frac{\partial \phi_{x_{1}}}{\partial \alpha} \frac{d \alpha}{d c_{1}}+\frac{\partial \phi_{x_{1}}}{\partial \beta} \frac{d \beta}{d c_{1}} & =1 \\
\frac{\partial \phi_{x_{2}}}{\partial \alpha} \frac{d \alpha}{d c_{1}}+\frac{\partial \phi_{x_{2}}}{\partial \beta} \frac{d \beta}{d c_{1}} & =0
\end{aligned}\right.
$$

Thus,

$$
\frac{d \alpha}{d c_{1}}=\frac{\partial \phi_{x_{2}} / \partial \beta}{\frac{\partial \phi_{x_{1}}}{\partial \alpha} \frac{\partial \phi_{x_{2}}}{\partial \beta}-\frac{\partial \phi_{x_{1}}}{\partial \beta} \frac{\partial \phi_{x_{2}}}{\partial \alpha}} \quad \text { and } \quad \frac{d \beta}{d c_{1}}=-\frac{\partial \phi_{x_{2}} / \partial \alpha}{\frac{\partial \phi_{x_{1}}}{\partial \alpha} \frac{\partial \phi_{x_{2}}}{\partial \beta}-\frac{\partial \phi_{x_{1}}}{\partial \beta} \frac{\partial \phi_{x_{2}}}{\partial \alpha}}
$$

Then, by (A24) and (A25), straightforward but rather tedious calculations yield

$$
\frac{d \alpha}{d c_{1}}=\frac{\gamma \alpha\left(\tau_{\theta}+\alpha^{2} \tau_{x}\right)\left(\tau_{\theta}+\beta^{2} x\right)}{2 \beta^{2} \tau_{\theta} \tau_{x}}>0
$$

and

$$
\frac{d \beta}{d c_{1}}=\frac{\gamma\left(\tau_{\theta}+\beta^{2} \tau_{x}\right)\left(\alpha^{2} \tau_{\theta}+\beta^{2}\left(2 \tau_{\theta}+\alpha^{2} \tau_{x}\right)\right.}{2 \beta^{3} \tau_{\theta} \tau_{x}}>0 .
$$

Turning to the case of $c_{2}$, we can immediately conclude due to symmetry that

$$
\frac{d \alpha}{d c_{2}}=\frac{\gamma\left(\tau_{\theta}+\alpha^{2} \tau_{x}\right)\left(\beta^{2} \tau_{\theta}+\alpha^{2}\left(2 \tau_{\theta}+\beta^{2} \tau_{x}\right)\right.}{2 \alpha^{3} \tau_{\theta} \tau_{x}}>0
$$

as well as

$$
\frac{d \beta}{d c_{2}}=\frac{\gamma \beta\left(\tau_{\theta}+\alpha^{2} \tau_{x}\right)\left(\tau_{\theta}+\beta^{2} x\right)}{2 \alpha^{2} \tau_{\theta} \tau_{x}}>0
$$

Thus, both $\alpha$ and $\beta$ are increasing in both cost parameters in equilibrium.

Furthermore, rearranging the system in (A32) with $\phi_{x_{1}}$ (resp., $\phi_{x_{2}}$ ) given by (A24) (resp., (A25)) yields a biquadratic equation in $\alpha$ and in $\beta$, respectively, that are symmetric. Then, direct computations show that these two equations only exhibit one pair of roots that satisfies $\alpha>0$ and $\beta>0$ simultaneously in this type of 
information acquisition equilibrium under certain conditions. It is given by

$\alpha\left(c_{1}, c_{2}\right)=\sqrt{-\frac{\left[e^{2 \gamma\left(c_{1}+c_{2}\right)}-e^{2 \gamma c_{1}}+\sqrt{\left(e^{2 \gamma c_{1}}-1\right)\left(e^{2 \gamma c_{2}}-1\right)} e^{2 \gamma c_{2}}\right] \tau_{\theta}}{\tau_{x}\left[e^{2 \gamma\left(c_{1}+c_{2}\right)}-\left(e^{2 \gamma c_{1}}+e^{2 \gamma c_{2}}\right)\right]}}$

and

$\beta\left(c_{1}, c_{2}\right)=\sqrt{-\frac{\left[e^{2 \gamma\left(c_{1}+c_{2}\right)}-e^{2 \gamma c_{2}}+\sqrt{\left(e^{2 \gamma c_{1}}-1\right)\left(e^{2 \gamma c_{2}}-1\right)} e^{2 \gamma c_{1}}\right] \tau_{\theta}}{\tau_{x}\left[e^{2 \gamma\left(c_{1}+c_{2}\right)}-\left(e^{2 \gamma c_{1}}+e^{2 \gamma c_{2}}\right)\right]}}$.

We immediately see that $(\alpha, \beta) \in \mathbb{R}_{++}^{2}$ requires

$$
\begin{array}{r}
e^{2 \gamma\left(c_{1}+c_{2}\right)}-\left(e^{2 \gamma c_{1}}+e^{2 \gamma c_{2}}\right)<0 \\
\Leftrightarrow c_{2}<\frac{1}{2 \gamma} \log \left[\frac{e^{2 \gamma c_{1}}}{e^{2 \gamma c_{1}}-1}\right] \equiv \bar{c}_{2}
\end{array}
$$

or by symmetry

$c_{1}<\frac{1}{2 \gamma} \log \left[\frac{e^{2 \gamma c_{2}}}{e^{2 \gamma c_{2}}-1}\right] \equiv \bar{c}_{1}$

Moreover, we already know that an information acquisition equilibrium of the form $\lambda_{1}^{*}>0$ and $\lambda_{2}^{*}>0$ requires $\gamma \alpha>\tau_{\epsilon}$ as well as $\gamma \beta>\tau_{\epsilon}$. In what follows, we investigate the case $\lambda_{1}^{*}>0$ and derive the respective results for $\lambda_{2}^{*}>0$ by symmetry. Note from (A33) that

$\alpha\left(0, c_{2}\right)=\sqrt{\frac{\left(e^{2 \gamma c_{2}}-1\right) \tau_{\theta}}{\tau_{x}}}$.

Since $\alpha$ is increasing in $c_{1}, \lambda_{1}^{*}>0$ holds for all $c_{1} \in\left(0, \bar{c}_{1}\right)$ if

$$
\begin{gathered}
\gamma \alpha\left(0, c_{2}\right)-\tau_{\epsilon} \geq 0 \\
\Leftrightarrow c_{2} \geq \frac{1}{2 \gamma} \log \left[1+\frac{\tau_{\epsilon}^{2} \tau_{x}}{\gamma^{2} \tau_{\theta}}\right]=\tilde{c} .
\end{gathered}
$$

Whenever $c_{2}<\tilde{c}, \lambda_{1}^{*}>0$ holds if and only if

$\gamma \alpha\left(c_{1}, c_{2}\right)-\tau_{\epsilon}>0$

Direct computations yield a quadratic inequality in $c_{1}$. It can be easily shown that the roots of the respective quadratic equation are given by $f\left(c_{2}\right)$ and $\bar{c}_{1}$. A direct 
comparison of $\bar{c}_{1}$ and $f\left(c_{2}\right)$ yields

$$
\begin{aligned}
\frac{1}{2 \gamma} \log \left[\frac{e^{2 \gamma c_{2}}}{e^{2 \gamma c_{2}}-1}\right] & \gtreqless \frac{1}{2 \gamma} \log \left[\frac{e^{2 \gamma c_{2}}\left[\left(e^{2 \gamma c_{2}}-1\right) \gamma^{4} \tau_{\theta}^{2}+\tau_{\epsilon}^{4} \tau_{x}^{2}\right]}{\left(e^{2 \gamma c_{2}}-1\right)\left(\gamma^{2} \tau_{\theta}+\tau_{\epsilon}^{2} \tau_{x}\right)^{2}}\right] \\
\Leftrightarrow c_{2} & \lesseqgtr \frac{1}{2 \gamma} \log \left[2+\frac{2 \tau_{\epsilon}^{2} \tau_{x}}{\gamma^{2} \tau_{\theta}}\right] \equiv \hat{c}>\tilde{c} .
\end{aligned}
$$

Thus, we can conclude that $\bar{c}_{1}>f\left(c_{2}\right)$ for all $c_{2}<\tilde{c}$. Furthermore, $\lambda_{1}^{*} \notin \mathbb{R}$ if $c_{1} \geq \bar{c}_{1}$. Recalling that $\alpha$ is increasing in $c_{1}, \lambda_{1}^{*}>0$ holds for

$$
\left\{\begin{array}{l}
\bar{c}_{1}>c_{1}>f\left(c_{2}\right) \equiv \frac{1}{2 \gamma} \log \left[\frac{e^{2 \gamma c_{2}}\left[\left(e^{2 \gamma c_{2}}-1\right) \gamma^{4} \tau_{\theta}^{2}+\tau_{\epsilon}^{4} \tau_{x}^{2}\right]}{\left(e^{2 \gamma c_{2}}-1\right)\left(\gamma^{2} \tau_{\theta}+\tau_{\epsilon}^{2} \tau_{x}\right)^{2}}\right] \text { if } c_{2}<\tilde{c} \\
c_{1} \in\left(0, \bar{c}_{1}\right) \text { if } c_{2} \geq \tilde{c}
\end{array}\right.
$$

Recall that $\beta$ is increasing in $c_{2}$. Then, by symmetry, we can immediately deduce that $\lambda_{2}^{*}>0$ holds for

$$
\left\{\begin{array}{l}
\bar{c}_{2}>c_{2}>f\left(c_{1}\right) \equiv \frac{1}{2 \gamma} \log \left[\frac{e^{2 \gamma c_{1}}\left[\left(e^{2 \gamma c_{1}}-1\right) \gamma^{4} \tau_{\theta}^{2}+\tau_{\epsilon}^{4} \tau_{x}^{2}\right]}{\left(e^{2 \gamma c_{1}}-1\right)\left(\gamma^{2} \tau_{\theta}+\tau_{\epsilon}^{2} \tau_{x}\right)^{2}}\right] \text { if } c_{1}<\tilde{c} \\
c_{2} \in\left(0, \bar{c}_{2}\right) \text { if } c_{1} \geq \tilde{c}
\end{array}\right.
$$

Next, we would like to unite the conditions in (A35) and (A36) by deducing the value range of $c_{2}$ in dependence of $c_{1}$ that simultaneously ensures $\lambda_{1}^{*}>0$ and $\lambda_{2}^{*}>0$ in equilibrium. We already know that $c_{2}<\tilde{c}$ and $c_{1}=f\left(c_{2}\right)$ would lead to $\lambda_{1}^{*}=0$. Furthermore, we have deduced that solving $c_{1}=f\left(c_{2}\right)$ for $c_{2}$ delivers two solutions, namely $g\left(c_{1}\right)$ and $h\left(c_{1}\right)$ (see the results from the third case).

Since $h\left(c_{1}\right)>\tilde{c}, c_{2}=h\left(c_{1}\right)$ can not yield $\lambda_{1}^{*}=0$. Thus, as $g\left(c_{1}\right)<\tilde{c}, g\left(c_{1}\right)$ is the unique value for $c_{2}$ that leads to $\lambda_{1}^{*}=0$. As $\alpha$ is increasing in $c_{2}, c_{2}>g\left(c_{1}\right)$ is a necessary condition for $\lambda_{1}^{*}>0$ to hold. As already derived, the upper bound of $c_{2}$ in an information acquisition equilibrium of the fourth case is $\bar{c}_{2}$.

Hence, the condition in (A35) can be written as $\bar{c}_{2}>c_{2}>g\left(c_{1}\right)$. Recall that $\bar{c}_{1}>f\left(c_{2}\right)$ for $c_{2}<\hat{c}$. Since $g\left(c_{1}\right) \in(0, \tilde{c})$ and $\tilde{c}<\hat{c}, g\left(c_{1}\right)<\hat{c}$ holds for all $c_{1} \in \mathbb{R}_{+}$. This leads to $\bar{c}_{2}>g\left(c_{1}\right)$ for all $c_{1} \in \mathbb{R}_{+}$.

Moreover, note that $g\left(c_{1}\right)$ is the inverse function of $f\left(c_{1}\right)$ for $c_{1}<\tilde{c}$. As $f(\bar{c})=\bar{c}, \lim _{c_{1} \rightarrow 0}$ $f\left(c_{1}\right)=\infty$, and $\lim _{c_{1} \rightarrow 0} g\left(c_{1}\right)=\tilde{c}, f\left(c_{1}\right)>g\left(c_{1}\right)$ holds for $c_{1} \in(0, \bar{c})$ and $g\left(c_{1}\right)>f\left(c_{1}\right)$ holds for $c_{1} \in(\bar{c}, \tilde{c})$. If $c_{1} \geq \tilde{c}, \lambda_{2}^{*}>0$ holds for all $c_{2} \in\left(0, \bar{c}_{2}\right)$ and the condition $c_{2}>f\left(c_{1}\right)$ becomes irrelevant. For $\lambda_{1}^{*}>0$ to be true in this case, $\bar{c}_{2}>c_{2}>g\left(c_{1}\right)$ still needs to be valid.

Eventually, we can conclude that an information acquisition equilibrium of the form 
$\lambda_{1}^{*}>0, \lambda_{2}^{*}>0$ requires

$\left\{\begin{array}{l}\bar{c}_{2}>c_{2}>f\left(c_{1}\right) \text { if } c_{1} \in(0, \bar{c}] \\ \bar{c}_{2}>c_{2}>g\left(c_{1}\right) \text { if } c_{1}>\bar{c}\end{array}\right.$

(A26), (A29), (A31), and (A37) together yield the conditions of the proposition.

Proof of Proposition 6. By (11), if $\lambda_{j}=0$, we have $I_{x_{j}}=0$. In this scenario, a rise in $\lambda_{i}$ does not affect the first term in (20). Thus, we get

$\frac{d \phi_{x_{i}}}{d \lambda_{i}}=-\frac{1}{2 \gamma} \mathbb{V a r}[\theta \mid P]\left(\frac{\partial \operatorname{Var}^{-1}[\theta \mid P]}{\partial I_{x_{i}}} \frac{d I_{x_{i}}}{d \lambda_{i}}\right)$

Building on (12), we can show that

$\frac{\partial \operatorname{Var}^{-1}[\theta \mid P]}{\partial I_{x_{i}}}=\frac{2 \tau_{\epsilon}^{2} \tau_{x}\left(1-I_{x_{i}}\right)}{\gamma^{2}\left[\left(1-I_{x_{i}}\right)^{2}+\left(1-I_{x_{j}}\right)^{2}\right]^{2}}$

and by symmetry

$\frac{\partial \operatorname{Var}^{-1}[\theta \mid P]}{\partial I_{x_{j}}}=\frac{2 \tau_{\epsilon}^{2} \tau_{x}\left(1-I_{x_{j}}\right)}{\gamma^{2}\left[\left(1-I_{x_{i}}\right)^{2}+\left(1-I_{x_{j}}\right)^{2}\right]^{2}}$.

Then, by (12), (A15), (A39), and $I_{x_{j}}=0,(\mathrm{~A} 38)$ becomes

$\frac{d \phi_{x_{i}}}{d \lambda_{i}}=-\frac{1}{2 \gamma} \frac{\tau_{\epsilon} \tau_{x}\left(1-I_{x_{i}}\right)\left\{2 \tau_{\epsilon}+\left(1-I_{x_{i}}\right)\left[\left(1-I_{x_{i}}\right)^{2}+1\right]^{2}\right\}}{\left[\left(1-I_{x_{i}}\right)^{2}+1\right]\left\{\gamma^{2} \tau_{\theta}\left[\left(1-I_{x_{i}}\right)^{2}+1\right]+\tau_{\epsilon}^{2} \tau_{x}\right\}}<0$.

If $\lambda_{j}>0$, both terms in (20) are affected by a rise in $\lambda_{i}$. By (A16), comparative statics analysis of the first term in (20) yields

$\frac{d \log \left[\tau_{\theta}+\frac{\tau_{\epsilon}^{2} \tau_{x}}{\gamma^{2}\left(1-I_{x_{j}}\right)^{2}}\right]}{d \lambda_{i}}=\frac{4 \tau_{\epsilon}^{3} \tau_{x}^{2}\left(1-I_{x_{i}}\right) I_{x_{j}}}{\gamma^{2}\left(1-4 I_{x_{1}} I_{x_{2}}\right)\left(1-I_{x_{j}}\right)^{2}\left[\gamma^{2}\left(1-I_{x_{j}}\right)^{2} \tau_{\theta}+\tau_{\epsilon}^{2} \tau_{x}\right]}$.

By inspecting (A41), it can be seen that the direction of the effect induced by a rise in $\lambda_{i}$ depends on the sign of $\Gamma$ (i.e., $1-4 I_{x_{1}} I_{x_{2}}$ ) and, hence, on the type of equilibrium traders coordinate on.

Comparative statics analysis regarding the second term in (20) yields

$\frac{d \log \left[\operatorname{Var}^{-1}[\theta \mid P]\right]}{d \lambda_{i}}=\mathbb{V} \operatorname{ar}[\theta \mid P]\left(\frac{\partial \operatorname{Var}^{-1}[\theta \mid P]}{\partial I_{x_{i}}} \frac{d I_{x_{i}}}{d \lambda_{i}}+\frac{\partial \operatorname{Var}^{-1}[\theta \mid P]}{\partial I_{x_{j}}} \frac{d I_{x_{j}}}{d \lambda_{i}}\right)$ 
By plugging (12), (A15), (A16), (A39), and (A40) into the equation above, we obtain

$\frac{d \log \left[\operatorname{Var}^{-1}[\theta \mid P]\right]}{d \lambda_{i}}=\frac{2 \tau_{\epsilon}^{3} \tau_{x}^{2}\left(1-I_{x_{i}}\right)\left[\left(1-I_{x_{i}}\right)^{2}+2\left(1-I_{x_{j}}\right)^{2} I_{x_{j}}\right]}{\gamma^{2} \chi\left(1-4 I_{x_{1}} I_{x_{2}}\right)\left(1-I_{x_{j}}\right)^{2}\left(\tau_{\theta} \gamma^{2} \chi+\tau_{\epsilon}^{2} \tau_{x}\right)}$,

where $\chi \equiv\left(1-I_{x_{i}}\right)^{2}+\left(1-I_{x_{j}}\right)^{2}$.

The sign of (A42) again depends on the equilibrium traders coordinate on. Putting (A41) and (A42) together eventually yields

$$
\begin{aligned}
\frac{d \phi_{x_{i}}}{d \lambda_{i}} & =\frac{1}{2 \gamma} \frac{d \log \left[\tau_{\theta}+\frac{\tau_{\epsilon}^{2} \tau_{x}}{\gamma^{2}\left(1-I_{x_{j}}\right)^{2}}\right]}{d \lambda_{i}}-\frac{1}{2 \gamma} \frac{d \log \left[\operatorname{Var}^{-1}[\theta \mid P]\right]}{d \lambda_{i}} \\
& =\frac{\left(1-I_{x_{i}}\right)^{3} \tau_{\epsilon}^{3} \tau_{x}^{2}\left\{\tau_{\theta} \gamma^{2}\left[2\left(1-I_{x_{i}}\right)^{2} I_{x_{j}}+\left(1-I_{x_{j}}\right)^{2}\left(4 I_{x_{j}}-1\right)\right]-\tau_{\epsilon}^{2} \tau_{x}\left(1-2 I_{x_{j}}\right)\right\}}{\gamma^{3}\left(1-4 I_{x_{1}} I_{x_{2}}\right)\left(1-I_{x_{j}}\right)^{2} \chi\left(\gamma^{2}\left(1-I_{x_{j}}\right)^{2} \tau_{\theta}+\tau_{\epsilon}^{2} \tau_{x}\right)\left(\tau_{\theta} \gamma^{2} \chi+\tau_{\epsilon}^{2} \tau_{x}\right)} .
\end{aligned}
$$

Thus, acquiring information about $x_{i}$ is a substitute (resp., complement) in the LIE if the numerator in (A43) is negative (resp., positive). By contrast, acquiring information about $x_{i}$ is a substitute (resp., complement) in the HIE if the numerator in (A43) is positive (resp., negative). Note that all given scenarios are plausible, since both $I_{x_{i}}$ and $I_{x_{j}}$ are independent of $\tau_{\theta}$.

Lastly, we investigate the influence of a change in $\lambda_{j}$ on $\phi_{x_{i}}$. By using the symmetric counterpart of (A15), we get

$$
\frac{d \log \left[\tau_{\theta}+\frac{\tau_{\epsilon}^{2} \tau_{x}}{\gamma^{2}\left(1-I_{x_{j}}\right)^{2}}\right]}{d \lambda_{j}}=\frac{2 \tau_{\epsilon}^{3} \tau_{x}^{2}\left(1-I_{x_{j}}\right)}{\gamma^{2}\left(1-4 I_{x_{1}} I_{x_{2}}\right)\left(1-I_{x_{i}}\right)^{2}\left[\gamma^{2}\left(1-I_{x_{j}}\right)^{2} \tau_{\theta}+\tau_{\epsilon}^{2} \tau_{x}\right]} .
$$

The term that captures the effect on price informativeness becomes

$$
\frac{d \log \left[\operatorname{Var}^{-1}[\theta \mid P]\right]}{d \lambda_{j}}=\frac{2 \tau_{\epsilon}^{3} \tau_{x}^{2}\left(1-I_{x_{j}}\right)\left[\left(1-I_{x_{j}}\right)^{2}+2\left(1-I_{x_{i}}\right)^{2} I_{x_{i}}\right]}{\gamma^{2} \chi\left(1-4 I_{x_{1}} I_{x_{2}}\right)\left(1-I_{x_{i}}\right)^{2}\left(\tau_{\theta} \gamma^{2} \chi+\tau_{\epsilon}^{2} \tau_{x}\right)}
$$

By (A44) and (A45), we finally obtain

$$
\begin{aligned}
\frac{d \phi_{x_{i}}}{d \lambda_{j}} & =\frac{1}{2 \gamma} \frac{d \log \left[\tau_{\theta}+\frac{\tau_{\epsilon}^{2} \tau_{x}}{\gamma^{2}\left(1-I_{x_{j}}\right)^{2}}\right]}{d \lambda_{j}}-\frac{1}{2 \gamma} \frac{d \log \left[\operatorname{Var}^{-1}[\theta \mid P]\right]}{d \lambda_{j}} \\
& =\frac{\tau_{\epsilon}^{3} \tau_{x}^{2}\left(1-I_{x_{j}}\right)\left\{\tau_{\theta} \gamma^{2}\left[\left(1-I_{x_{i}}\right)^{2}+2\left(1-I_{x_{j}}\right)^{2}\left(1-I_{x_{i}}\right)\right]+\tau_{\epsilon}^{2} \tau_{x}\left(1-2 I_{x_{i}}\right)\right\}}{\gamma^{3}\left(1-4 I_{x_{1}} I_{x_{2}}\right) \chi\left[\gamma^{2}\left(1-I_{x_{j}}\right)^{2} \tau_{\theta}+\tau_{\epsilon}^{2} \tau_{x}\right]\left(\tau_{\theta} \gamma^{2} \chi+\tau_{\epsilon}^{2} \tau_{x}\right)} .
\end{aligned}
$$


Consequently, acquiring information about different noise components is a substitute (resp., complement) in the LIE if the numerator in (A46) is negative (resp., positive). Inversely, acquiring information about different noise components is a substitute (resp., complement) in the HIE if the numerator in (A46) is positive (resp., negative). Again all scenarios can occur since the endogenous values of both trading intensities do not vary with $\tau_{\theta}$.

\section{References}

Avdis, E. (2016). Information Tradeoffs in Dynamic Financial Markets. Journal of Financial Economics, 122(3), 568-584.

Barber, B. M., Odean, T., \& Zhu, N. (2006). Do Noise Traders Move Markets? Working Paper.

Black, F. (1986). Noise. The Journal of Finance, 41(3), 528-543.

Cespa, G. \& Vives, X. (2012). Dynamic Trading and Asset Prices: Keynes vs. Hayek. The Review of Economic Studies, 79(2), 539-580.

Cespa, G. \& Vives, X. (2015). The Beauty Contest and Short-Term Trading. The Journal of Finance, 70(5), 2099-2154.

Demange, G. \& Laroque, G. (1995). Private Information and the Design of Securities. Journal of Economic Theory, 65(1), 233-257.

Dickson, L. E. (1914). Elementary Theory of Equations. Boston: F. H. Gilson Company.

Farboodi, M. \& Veldkamp, L. (2020). Long-Run Growth of Financial Data Technology. The American Economic Review, 110(8), 2485-2523.

Ganguli, J. V. \& Yang, L. (2009). Complementarities, Multiplicity, and Supply Information. Journal of the European Economic Association, 7(1), 90-115.

Garcia, D. \& Strobl, G. (2011). Relative Wealth Concerns and Complementarities in Information Acquisition. The Review of Financial Studies, 24(1), 169-207.

Gennotte, G. \& Leland, H. (1990). Market Liquidity, Hedging, and Crashes. The American Economic Review, 999-1021.

Goldstein, I., Li, Y., \& Yang, L. (2014). Speculation and Hedging in Segmented Markets. The Review of Financial Studies, 27(3), 881-922. 
Goldstein, I. \& Yang, L. (2015). Information Diversity and Complementarities in Trading and Information Acquisition. The Journal of Finance, 70 (4), 1723-1765.

Grossman, S. J. \& Stiglitz, J. E. (1980). On the Impossibility of Informationally Efficient Markets. The American Economic Review, 70(3), 393-408.

Hellwig, M. F. (1980). On the Aggregation of Information in Competitive Markets. Journal of Economic Theory, 22(3), 477-498.

Hirshleifer, D., Subrahmanyam, A., \& Titman, S. (1994). Security Analysis and Trading Patterns when some Investors Receive Information before Others. The Journal of Finance, 49(5), 1665-1698.

Manzano, C. \& Vives, X. (2011). Public and Private Learning from Prices, Strategic Substitutability and Complementarity, and Equilibrium Multiplicity. Journal of Mathematical Economics, 47(3), 346-369.

Marmora, P. \& Rytchkov, O. (2018). Learning about Noise. Journal of Banking \&6 Finance, 89, 209-224.

Medrano, L. \& Vives, X. (2004). Regulating Insider Trading When Investment Matters. Review of Finance, 8(2), 199-277.

Rahi, R. \& Zigrand, J.-P. (2014). Information Aggregation in a Competitive Economy. London School of Economics Working Paper.

Veldkamp, L. L. (2006). Media Frenzies in Markets for Financial Information. American Economic Review, 96(3), 577-601.

Vives, X. (2008). Information and Learning in Markets: The Impact of Market Microstructure. Princeton/Oxford: Princeton University Press. 